\title{
Induced Bragg Gratings in Optical Fibers and Waveguides Using an Ultrafast Infrared Laser and a Phase Mask
}

\author{
Stephen J. Mihailov, Dan Grobnic, Christopher W. Smelser, Ping Lu, \\ Robert B. Walker, and Huimin Ding \\ Optical Communications and Electrophotonics Group, Communications Research Centre Canada, 3701 Carling Avenue, \\ Ottawa, Ontario, Canada K2H $8 \mathrm{~S} 2$ \\ Correspondence should be addressed to Stephen J. Mihailov, stephen.mihailov@crc.ca
}

Received 26 March 2008; Accepted 22 April 2008

Recommended by Saulius Juodkazis

\begin{abstract}
Since its development in 2003, the technique of Bragg grating inscription in optical fibers and waveguides with ultrafast infrared radiation and a phase mask has proven to be as simple as the standard UV-laser grating writing techniques but far more versatile. The ultrafast IR laser-based process allows for the creation of grating structures in glassy and crystalline materials that are not typically UV photosensitive. In this article, we will review the studies that have been performed at the Communications Research Centre Canada on the grating formation processes as well as applications of the ultrafast laser technique to fabricate gratings in various optical fibers and waveguides.
\end{abstract}

Copyright (C) 2008 Stephen J. Mihailov et al. This is an open access article distributed under the Creative Commons Attribution License, which permits unrestricted use, distribution, and reproduction in any medium, provided the original work is properly cited.

\section{Introduction}

High-power femtosecond (fs) lasers systems are being used extensively for laser-material processing of glassy materials in order to fabricate microfluidic and photonic devices. The ultrahigh peak power infrared (IR) radiation generated by many of these systems has been used to induce large index changes in bulk glasses for the fabrication of imbedded waveguides and surface structures $[1,2]$. In glass optical fiber, femtosecond laser systems have been used to induce large index changes and fabricate long-period fiber grating structures in a step-and-repeat fashion $[3,4]$.

The process of induced index change in bulk glasses resulting from femtosecond-IR laser exposure is thought to result from a multiphoton absorption/ionization process resulting in material compaction and/or defect formation depending on the intensity of the exposure [1]. Above the ionization threshold intensity Ith, multiphoton ionization (MPI) induced dielectric breakdown likely results in localized melting and material compaction causing an index change that is permanent up to the glass transition temperature $t_{g}$, of the material. The value of Ith is materialdependent.

Below Ith, another regime of induced index change has been observed that can be erased by annealing with temperatures below the material $t_{g}$ [5]. In this regime, multiphoton absorption likely results in defect formation similar to that seen for ultraviolet-(UV-) induced index changes in photosensitive germanium doped silica glasses.

Laser induced index change is the key feature of most fiber Bragg grating (FBG) manufacturing. Typically FBGs are made by side exposure of the UV-sensitive fiber core to a spatially modulated UV laser beam. The modulated UV beam is typically created by interfering two or more UV beams using either a bulk interferometer or a zeroorder-nulled phase mask [6]. For femtosecond systems, interferometric setups for generating beam modulation are nontrivial to align since path lengths of the interfering beams need to be matched to within the spatial location of the femtosecond-pulse [2], which for a 120-femtosecond pulse would be $\sim 36 \mu \mathrm{m}$. The phase mask technique solves the alignment problem since the optical path lengths of the 
generated beams are automatically matched for an incoming beam that is at normal incidence to the mask.

Femtosecond UV laser inscriptions of fiber gratings using silica phase masks have been performed by a number of research groups [7-10]. Typically, silica-based fibers are placed in close proximity to the mask, and large index changes have been generated in suitably UV photosensitive waveguides. A limitation of this technique however is that lower pulse intensities need to be employed since high nonlinear absorption and group velocity dispersion would otherwise occur within the mask. Such nonlinear absorption reduces the amount of light transmitted through the mask that is available to induce an index change in the fiber and simultaneously degrades the phase mask with time.

Combining the ultrafast IR laser and the phase mask approach, we successfully demonstrated the efficient fabrication of retroreflecting FBGs in standard telecom and pure silica core single mode fibers $[11,12]$. The technique has proven to be as simple as the standard UV-laser writing technique but is far more versatile as grating inscription is not limited to materials that are traditionally UV-photosensitive such as Ge-doped silica. Using the femtosecond-IR laser and the phase mask, we have successfully inscribed high indexmodulation grating structures in pure silica core fibers [12], borosilicate glass waveguides [13], reverse proton exchange $\mathrm{LiNb}_{3}$ waveguides [14], sapphire optical fiber [15, 16], fluoride glass fibers [17], actively doped silica fibers [18], phosphate glass fibers [19], and pure silica photonic crystal fibers and tapers [20]. In this paper, we will summarize our investigations into the processes of ultrafast IR laser-induced FBGs with a phase mask along with many of the applications that have arisen from the use of this technique, such as direct fabrication of gratings through protective fiber coatings, fabrication of fiber laser cavities, and the fabrication of hightemperature-stable grating sensors.

\section{Phase Mask Design}

The phase masks used in these studies are similar to standard all-silica phase masks used for UV laser FBG inscription however the etch depth is optimized for operation in the near IR $(800 \mathrm{~nm})$ as opposed to the UV. Standard UV masks used for fabrication of C-band FBGs typically have mask pitches $\Lambda_{m}$ of $\sim 1.07 \mu \mathrm{m}$ with good zero-order transmission suppression. The majority of the transmitted energy then couples into the \pm 1 orders generated by the mask. Even when optimized for operation at $800 \mathrm{~nm}$, a mask pitch of $1.07 \mu \mathrm{m}$ has considerable coupling of the incident energy into the zero-order due to the proximity of the irradiating wavelength to $\Lambda_{m}[11,12]$. Nevertheless, high-quality gratings can be inscribed using standard mask pitches as will be described below.

In addition to standard mask pitches, phase masks were fabricated with $\Lambda_{m}$ values that would produce higher-order Bragg resonances $\lambda_{B}$. At $\lambda_{B}$, the grating pitch in the fiber, $\Lambda_{G}$, is defined by $m \lambda_{B}=n_{\text {eff }} \Lambda_{G}$ where $m$ is the order number and $n_{\text {eff }}$ is the effective index of the fiber. As the process for ultrafast IR laser-induced index change is a nonlinear one, it was thought to be likely that higher-order

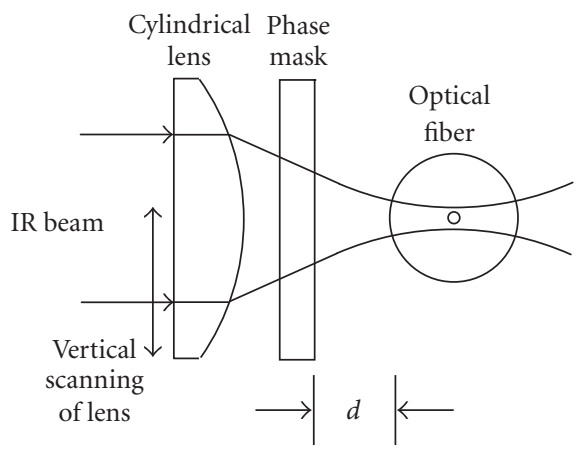

FIGURE 1: Schematic representation of lens-fiber-phase mask geometry for waveguide exposures.

mask pitches would easily produce Bragg resonances in the telecom band. Four phase masks were then produced by ebeam lithography with different pitches such that higherorder Bragg resonances occurred at $1550 \mathrm{~nm}$ within standard telecom Corning SMF-28 fiber, namely 4.284, 3.213, 2.142, and $1.071 \mu \mathrm{m}$. Gratings with high index modulations, $\Delta n$, were easily written with each phase mask. Gratings written with the higher-order pitch phase masks (i.e., $>1.07 \mu \mathrm{m}$ ) had the additional advantage of having grating structures that were directly observable under a standard optical microscope.

\section{Experimental Conditions}

In these studies, Fourier transform limited 125 femtosecond duration laser pulses from a regeneratively amplified Ti: sapphire laser operating at $800 \mathrm{~nm}$ were used to illuminate optical fibers and waveguides by passing the irradiating beam through a cylindrical focusing lens and a phase mask as shown in Figure 1. Gaussian beam diameters incident on the focusing lens were typically $6.4 \mathrm{~mm}$. Depending on the study that was being performed, pulse energies were varied between 0.2 and $2 \mathrm{~mJ} /$ pulse, repetition rates were varied from 1 to $1000 \mathrm{~Hz}$, and various cylindrical focusing lenses were used ( $f=12$ to $160 \mathrm{~mm}$ ). When tight focusing geometries were used, it was found that vertical scanning of the irradiating beam across the waveguide normal to its axis eased alignment tolerances of the beam with respect to the waveguide core as well as allowed for the fabrication of many interesting grating structures. Typically, the incident beam was centered onto the fiber core and then scanned vertically over the fiber cross-section using a piezoactuated translation stage with $\mathrm{a} \pm 10 \mu \mathrm{m}$ travel and a 20 -second period. The fiberphase mask distance $d$ also proved to be a very important parameter as it would allow for the writing of pure two-beam interference gratings with a phase mask, even one possessing poor zero-order suppression. Grating spectra were typically measured using an $\mathrm{Er}^{+}$white light source and an optical spectrum analyzer or a tunable C-L band laser.

Annealing experiments of grating structures were typically performed in a Lindberg tube furnace equipped with an internal quartz tube of $2.54 \mathrm{~cm}$ diameter centered within the furnace tube. Gratings were loosely placed in the inner 
tube so that no external stresses were applied to the grating. The experiments were performed in ambient air, and the heating rate was limited to $10^{\circ} \mathrm{C} / \mathrm{min}$. A swept tunable laser system was used to continually measure the grating spectral responses.

\section{Ultrafast Laser-Induced Grating Processes}

\subsection{Phase Mask-Order Interference: Order Walk Off}

Ultrafast laser pulses interact differently with phase masks as compared to nanoseconds pulses or continuous wave CW sources. The spatial localization of the ultrafast pulse envelope incident on the mask is small $(\sim 40 \mu \mathrm{m}$ for a 125 femtosecond pulse). As the diffracted pulses produced by the mask propagate away from the mask surface, the propagation distance of each pulse, when projected onto the optical axis normal to the mask, appears different, as is shown in Figure 2(a). The diffracted pulse envelopes have different arrival times at a given distance $d$ normal to the mask. For sufficiently large $d$, the diffracted-order pairs $(0$, $\pm 1, \pm 2$, etc.) no longer overlap resulting in a diffractedorder walk-off effect (see Figure 2(b)). For a mask with $\Lambda_{m}=3.213 \mu \mathrm{m}$ and a 125 femtosecond pulse, the spatial separation of the \pm 1 order from the zero and \pm 2 orders would occur at fiber-mask distances $d>1.3 \mathrm{~mm}$. As the induced index change using ultrafast IR radiation is highly nonlinear and highly localized within the target material, small $d$ values (i.e., near the mask) produce multiple-order complex interference field patterns within the waveguide [21]. For large $d$ values, the two-beam interference fringe patterns, caused by the diffracted-order walk-off effect, can be produced inside the waveguide with only the high-energy \pm 1 orders from the mask (see Figure 2(b)) [22]. A clear advantage of the walk-off effect is that for short pulses, the phase mask need not be zero-order suppressed to produce a two-beam interference pattern. Although not investigated here, it is likely that this remote writing process would also be advantageous for grating inscription with femtosecond UV sources. By using a tight focusing arrangement and a large displacement between the phase mask and fiber, the power densities and hence nonlinear absorption in the phase mask could be significantly reduced thus prolonging the life of the phase mask and allowing for higher power densities of UV light to interact with the fiber.

\subsection{Type I-IR Versus Type II-IR}

With the application of the order walk-off effect and vertical scanning of the beam across the waveguide axis, fabrication of gratings could be performed in a controlled way allowing for the study of grating formation processes below and above the ionization threshold Ith. For femtosecond pulses interacting near the phase mask, the complex interference field resulting from multiple beam overlap, had high-peak intensities that would create gratings through an ionization process likely accentuated by self-focusing. This process

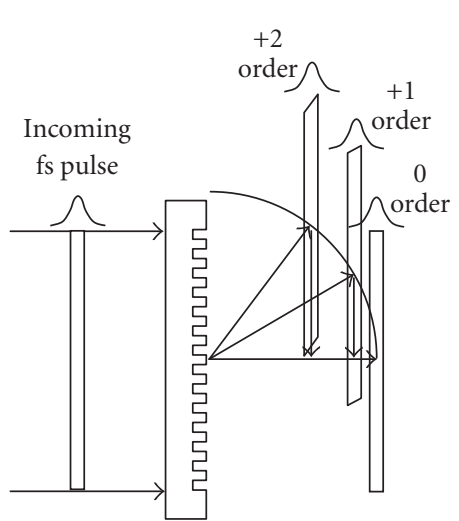

(a)

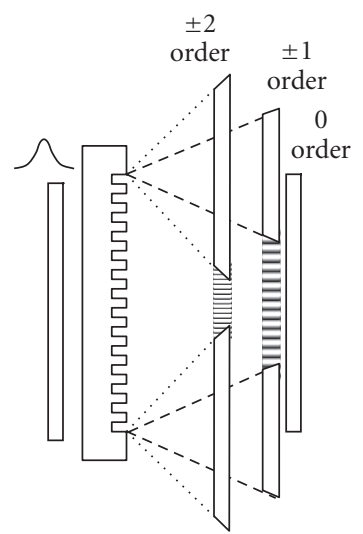

(b)
FIGURE 2: Schematic of diffractive order walk-off (a) pulse envelope projection normal to phase mask, (b) two-beam interference from each order pair.

produced gratings with the same pitch as the mask that were stable up to the glass transition temperature of the fiber. Sudrie et al. defined this intensity regime for index change in bulk silica glasses as type II [5]. In order to avoid confusion with type II UV-induced gratings, we defined this FBG formation regime as being type II-IR [23]. At greater distances from the mask, for the same pulse energy, the generated two-beam interference pattern had reduced peak intensities that did not traverse the threshold value for type II formation. Grating structures with high $\Delta n$ 's were created after a more lengthy exposure that had a grating pitch that was half that of the mask.

To study the stability of the $\Delta n$ of the gratings written in the type I-IR and II-IR regimes, an isochronal annealing study was performed where the FBG temperature was increased in $100^{\circ} \mathrm{C}$ increments and held at each temperature for one hour. For comparison, a type I UV-induced FBG was inscribed in $\mathrm{H}_{2}$-loaded $S M F-28$ fiber $\left(3000 \mathrm{psi}, 85^{\circ} \mathrm{C}\right.$, 24 hours) using a frequency doubled argon-ion laser and a phase mask which produced a $\Delta n=5 \times 10^{-4}$. The estimated initial $\Delta n$ for the femtosecond IR gratings was $1.5 \times 10^{-3}$. In Figure 3, the short-term performance of the UV and femtosecond IR FBGs with respect to temperature is presented where the initial $\Delta n$ of each of the FBGs is normalized to 1 . With increasing temperature, the $\Delta n$ of the UV grating, denoted by the circles, decreases and completely disappears when the grating temperature approaches $1000^{\circ} \mathrm{C}$. The annealing properties of the type I-IR FBG, denoted by black squares in Figure 3, are very similar to those of the type I UV gratings. For the fs type II-IR FBG, the reflectivity increased slightly up to $500^{\circ} \mathrm{C}$ and then remained stable. These annealing results suggest that the type I-IR gratings are due to a highly nonlinear defect formation process resulting from multiphoton absorption while the type II-IR gratings are "damage" gratings resulting from a multiphoton ionization process. The increase in the $\Delta n$ of the type II-IR grating with temperature is likely the result of annealing of less stable index change that occurs in the 
less intense trough regions of the interference field. As the intensities in these regions are below $I$ th, the index change is the result of a type I-IR process and is erased at elevated temperatures.

\subsection{Type I-IR Scaling Behavior}

Compared to techniques that measure femtosecond IRinduced index changes in bulk glasses, the technique of Bragg grating inscription in existing waveguides for measurement of index change is very sensitive thus allowing for the detection of index changes as small as $1 \times 10^{-6}$. A series of experiments were performed in Ge-doped $S M F-28$ fiber using the setup shown in Figure 1 in order to determine the scaling behavior of the $\Delta n$ growth of a type I-IR grating as a function of the interference field intensity, where peak field intensity was directly related to the pulse energy [23]. The $6.4 \mathrm{~mm}$ diameter beam from the IR-laser was focused with a $f=30 \mathrm{~mm}$ focal length through the $\Lambda_{m}=3.21 \mu \mathrm{m}$ phase mask onto the fiber. The fiber was placed at distance $d=$ $5 \mathrm{~mm}$ behind the mask to ensure two-beam interference. The pulse repetition rate of the laser was kept at $100 \mathrm{~Hz}$. Several FBGs were then fabricated using the different pulse energies of $850,900,950,1000,1100$, and $1200 \mu \mathrm{J}$. The transmission loss at $\lambda_{B}$ for each grating was continuously monitored. The spectra were then modeled using a commercial FBG software package.

The growth of $\Delta n$ as a function of exposure time and pulse energy is presented in Figure 4(a) as well as the scaling behavior as a function of energy, which is presented in Figure 4(b). Linear regression of the logarithm of the scaling behavior versus the logarithm of the pulse energy yields a slope of 5 , meaning that the absorption process involved in the index growth scales with the fifth power of the peak intensity of the interference field $I^{5}$. A fifth power dependency on intensity suggests that the absorption process is a 5 -photon one.

The $800 \mathrm{~nm}$ wavelength or $1.55 \mathrm{eV}$ photon energy of the ultrafast IR source is far removed from the typical bandgap of Ge-doped silica, which is thought to be $\sim 7.1 \mathrm{eV}$ [24]. A 5-photon absorption process amounting to $7.75 \mathrm{eV}$ has sufficient energy to bridge the $7.1 \mathrm{eV}$ bandgap of the Gedoped silica. Similar annealing behavior between the type IIR and type I-UV gratings also suggests that a similar defect and/or compaction related mechanism of induced index change may be occurring in the type I-IR FBGs.

\subsection{Hydrogen Loading}

The possibility that a similar mechanism of induced index change was occurring in the Ge-doped fibers for both type I-UV and type I-IR FBGs suggested that fiber photosensitization processes used for UV exposures such as high Ge-core content of the waveguide or hydrogen loading [25] might also enhance type I-IR grating formation in Ge-doped fibers. As femtosecond-IR exposures of Ge-doped bulk silica generate $\mathrm{GeE}$ ' and germanium-oxygen-deficient centers [1], FBG formation threshold intensity reduction with $\mathrm{H}_{2}$-loading may result from a similar mechanism as seen in UV-induced

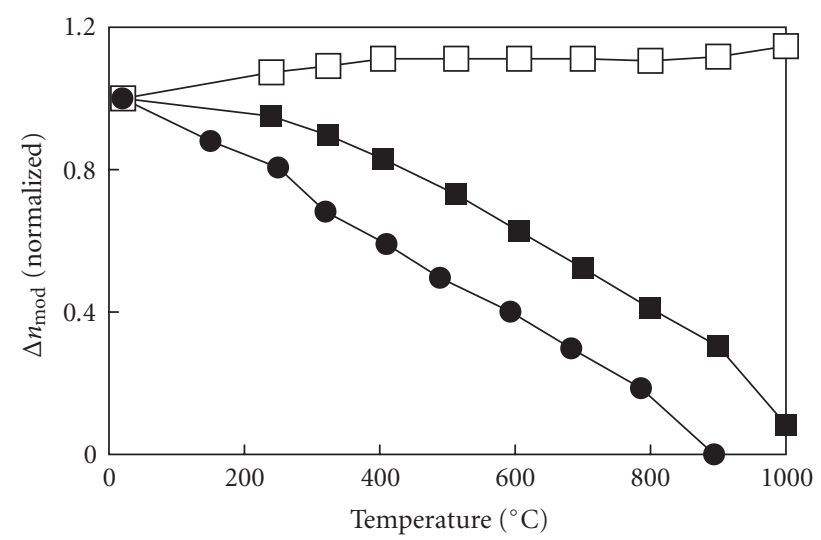

FIGURE 3: Short-term annealing of type II-IR (white square), type I-IR (black square), type I-UV (black cirlce), grating held at each temperature for 1 hour.

index changes. Increased UV-photosensitivity due to $\mathrm{H}_{2}$ loading of Ge-doped silica is likely due to higher formation rates of GeE' centers through photoinduced reactions of $\mathrm{H}_{2}$ with $\mathrm{Ge}_{-} \mathrm{SiO}_{2}$ [26]. This may be the case in the femtosecondIR regime as well. Another series of experiments were then performed to determine if there was a variation in the threshold pulse energies needed to induce a $\Delta n$ in standard telecom Ge-doped fibers when loaded with high-pressure hydrogen. For comparison, measurements of the threshold intensity for grating inscription in unloaded Ge-doped fibers as well as loaded and loaded pure silica core single mode fibers were also performed [27]. Fibers were $\mathrm{H}_{2}$-loaded at $85^{\circ} \mathrm{C}, 3000$ psi for 24 hours.

While no variation in the intensity threshold for grating formation was observed in either the $\mathrm{H}_{2}$-loaded or unloaded all-silica core single mode fiber, the threshold intensity in the case of the SMF-28 fiber was reduced by a factor of 3 . For comparison, isochronal annealing studies were preformed on UV-induced and ultrafast-IR-induced FBGs written in $\mathrm{H}_{2}$-loaded $S M F-28$ fiber. Temperatures were increased in $100^{\circ} \mathrm{C}$ increments and allowed to stabilize for one hour. The annealing curves of low- and high-reflectivity gratings induced with either a frequency doubled UV $\mathrm{Ar}^{+}$laser or the femtosecond-IR laser show similar responses to temperature (see Figure 5). For the FBG's reported in [27], no broadband IR absorption due to the formation of Ge-OH defects had been observed. In subsequent experiments however that were not previously reported, the induction of high $\Delta n=5 \times$ $10^{-3}$ in $\mathrm{H}_{2}$-loaded $S M F-28$ fibers did in fact result in the formation of the $\mathrm{Ge}-\mathrm{OH}$ absorption band in the $1400 \mathrm{~nm}$ range suggesting that the photosensitivity enhancement due to hydrogen for ultrafast IR may have similar mechanisms as those that occur for UV exposures.

\subsection{Modeling Nonlinear Index Change: the Rouard Method}

Typically, the modeling of UV-induced gratings using coupled mode theory, the matrix method or Rouard's method 


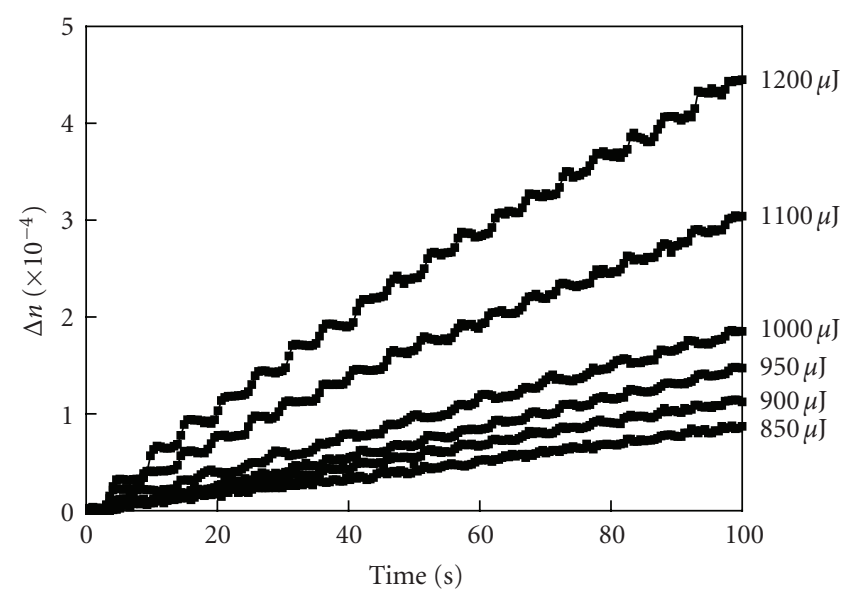

(a)

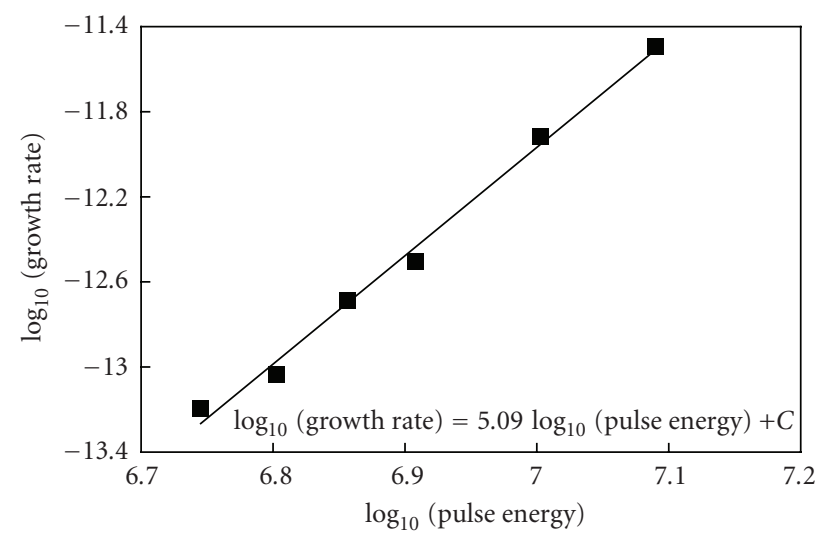

(b)

FIGURE 4: (a) Growth of the index modulation as a function of time and pulse energy. (b) Scaling behavior of the $\Delta n$ growth rate as function of energy.

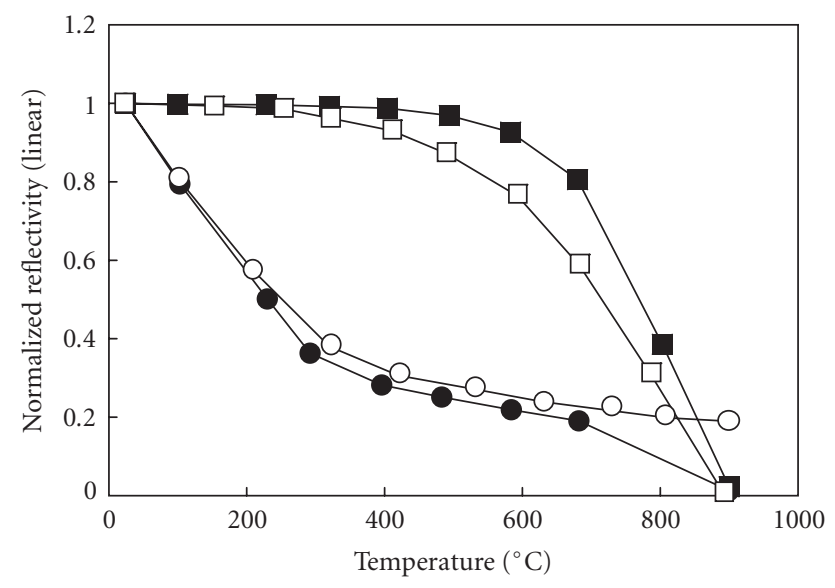

FIGURE 5: Isochronal annealing curves of low- (circles, $\Delta n \sim 10^{-5}$ ) and high- (squares, $\Delta n \sim 10^{-3}$ ) reflectivity FBGs written in $\mathrm{H}_{2}$ loaded SMF-28 fiber with CW UV laser light (white shape) or femtosecond-IR laser light (black shape).

assumes that the $\Delta n$ is sinusoidal in nature [28]. As the process for type I-IR-induced index change has been shown to be highly nonlinear, this assumption is no longer valid. In order to model the type I-IR FBG spectral responses the nonsinusoidal nature of the induced $\Delta n$ needs to be taken into account.

Rouard's method is an effective technique for modeling an arbitrary index profile [29-31] in a waveguide. By using Rouard's method to model ultrafast IR FBG grating responses, the nonsinusoidal nature of the type I-IR $\Delta n$ was taken into account by subdividing individual fringe planes into a series of Fresnel reflections [32]. This technique proved to be quite powerful as it automatically generated for example, higher-order Bragg resonances when a nonsinusoidal $\Delta n$ was considered. Such higher-order Bragg resonances occur when the $\Delta n$ deviates from a sinusoidal profile [33]. The model also provided subtle insight into variations of growth rates of $\Delta n$ as a function of incident intensity. For peak fringe intensities in $S M F-28$ fiber that were above the threshold for grating formation but well below those that would produce a type II-IR grating, the spectral response was most accurately modeled using an $I^{6}$ scaling of the $\Delta n$ (see Figure 6) [32].

For fringe intensities that were approaching the type IIIR threshold, saturation in the growth rate of the $\Delta n$ is observed which results in a more "top-hat" like modulation profile. The spectral response was most accurately modeled using a saturation function for the normalized $\Delta n$ where

$$
\Delta n_{\mathrm{norm}}=\frac{I^{6}}{C+I^{6}},
$$

and $C$ is a constant equaling 0.05 . Figure 7 presents the experimental result along with modeled response [32]. Limiting behavior and intensity clamping are common in highly nonlinear absorption processes and may be responsible for this observed saturation effect [34].

\subsection{High-Order Spectral Response Characteristics}

We have shown that the five-photon absorption process associated with the type I-IR gratings results in a $\Delta n$ profile that is nonsinusoidal. Unlike UV laser-induced gratings, which in the absence of saturation of the index change have only one Bragg resonance, the type I-IR gratings possess a number of higher-order Bragg resonances [35].

If the index modulation along the grating in the waveguide no longer follows a sinusoidal profile, $\Delta n(z)$ can be expanded as a Fourier series $[33,36]$ :

$$
\Delta n(z)=\frac{\Delta n_{0}}{2}+\sum_{m=1}^{\infty} \Delta n_{m} \cos \left(\frac{2 m \pi z}{N \Lambda_{G}}\right),
$$

where $m$ is the order number, $N \Lambda_{G}$ is the grating periodicity in the waveguide that is an integer number $N$ multiplied by 


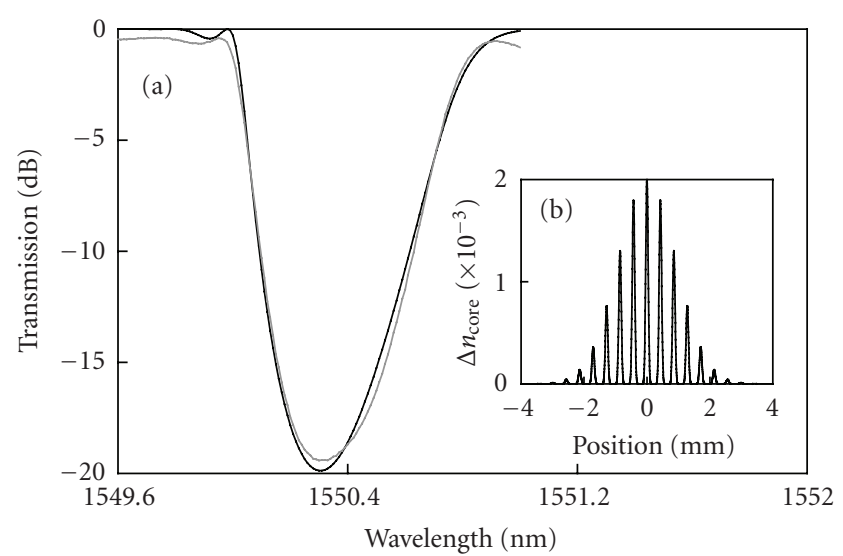

Figure 6: Comparison of modeled (black) and experimental (gray) spectra for an irradiation intensity below the type I-IR to type II-IR transition $\left(2.7 \times 10^{13} \mathrm{~W} / \mathrm{cm}^{2}\right)$. Inset is the $\Delta n$ profile used to model the spectrum; fringe spacing is not to scale in figure.

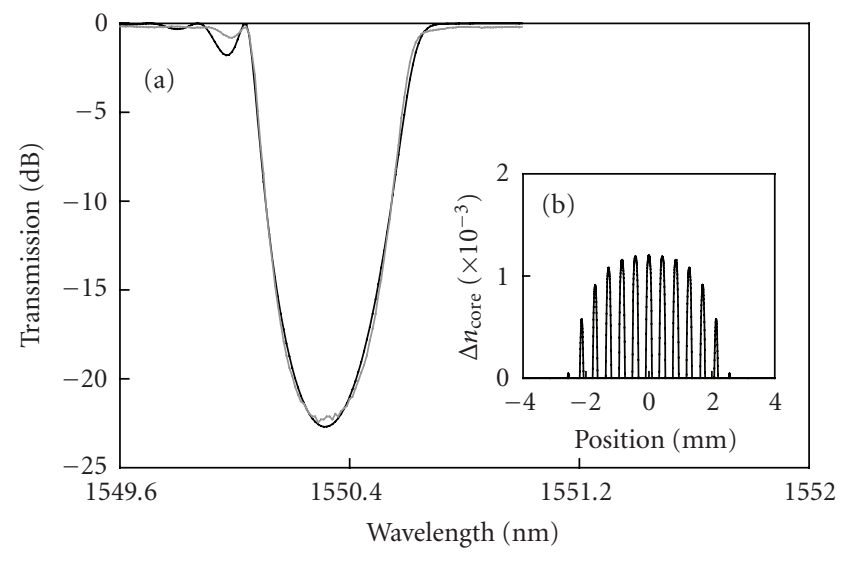

Figure 7: Comparison of experimental (black) and modeled (gray) spectra for an irradiation intensity approaching the type I-IR to type II-IR transition $\left(4 \times 10^{13} \mathrm{~W} / \mathrm{cm}^{2}\right)$. Inset is the index modulation profile used to model the spectrum; fringe spacing is not to scale in figure.

the first-order grating pitch. $\Lambda_{G}$ and $\Delta n_{m}$ are the Fourier coefficients of order $m . \Delta n_{0}$ is the index change averaged over the length of the grating and is often associated with the wavelength shift of the Bragg resonance $\lambda_{B}$.

In [36], the evaluation of the Fourier components associated with each grating mask was determined for the order resonance that occurred in the $1550 \mathrm{~nm}$ wavelength range for both unloaded and $\mathrm{H}_{2}$-loaded $S M F-28$ telecommunication fiber, for example the $1550 \mathrm{~nm}$ resonance of a grating made with the $4.28 \mu \mathrm{m}$ phase mask using the two-beam interference technique corresponds to the $\Delta n_{4}$ Fourier component. By monitoring the wavelength shift that occurred while inscribing gratings of a specific reflectivity with each of the phase masks pitched 1.071, 2.142, 3.213, and $4.284 \mu \mathrm{m}$, respectively, the relative amplitudes for the Fourier coefficients were determined (see Figure 8).

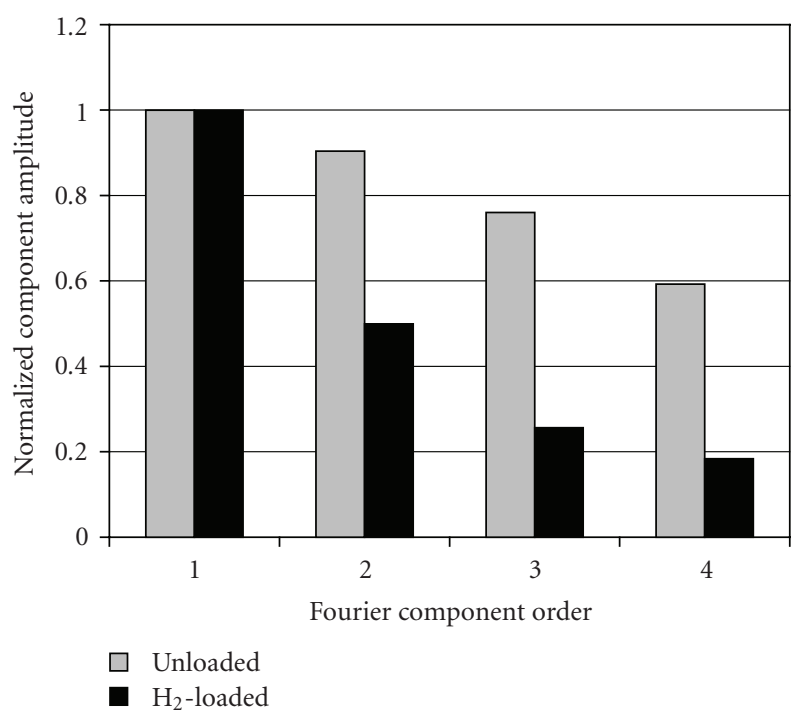

Figure 8: Comparison of the Fourier components for the pristine and hydrogen-loaded $S M F-28$ fiber. The magnitude of the firstorder component has been set to 1 .

Using pulse intensities that were below the threshold for grating formation in unloaded fiber, the Fourier components of gratings written in $\mathrm{H}_{2}$-loaded $S M F-28$ fiber are consistent with those that would be achieved through a 5-photon distortion of the sinusoidal modulation. For gratings in unloaded fiber written with intensities mid-way between the type I-IR threshold and the type II-IR threshold, the Fourier components are consistent with a modulation profile that is squarer.

For gratings written in the type II-IR regime, the induced index change is no longer the result of a multiphoton absorption process but a multiphoton ionization process resulting in localized damage. Using a Corning 980 Flexcorefiber with a cutoff wavelength of $980 \mathrm{~nm}$, and the $4.28 \mu \mathrm{m}$ pitched phase mask, the induced type II-IR grating generates a large number of higher-order Bragg resonances in the 1000-1800 nm range, many with almost equal coupling; see Figure 9. This suggests that in the type II-IR regime the induced index modulation has a much more square profile than does the 5-photon absorption related profile.

\section{Applications of Ultrafast Induced Bragg Gratings in Optical Fiber and Aaveguides}

\subsection{Cladding Mode Suppression}

An important application of FBG technology is for high reflectivity narrowband reflectors in wavelength-division multiplexing (WDM) systems of optical networks. Such FBGs typically suffer from an out-of-band loss on the short wavelength side of the Bragg resonance which is due to strong coupling from the forward propagating core mode $\left(\mathrm{LP}_{01}\right)$ into discrete backward-propagating cladding modes [37]. Cladding mode coupling is caused by a mismatch in the overlap between the $\mathrm{LP}_{01}$ mode and the fiber core in 


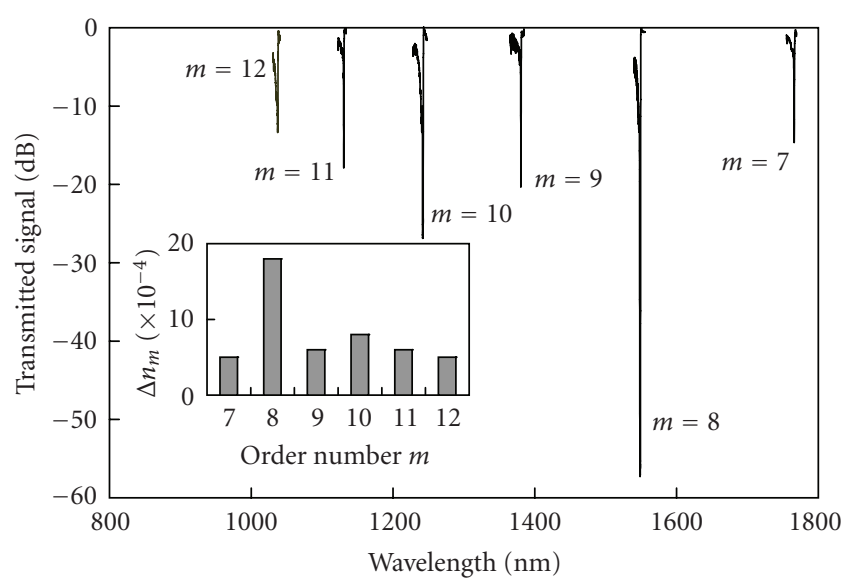

FIGURE 9: Transmission spectrum of a type II-IR grating written in $980 \mathrm{~nm}$ cutoff single mode fiber with the $4.28 \mu \mathrm{m}$ pitched phase mask. Inset depicts the calculated index modulations of each of the diffracted orders assuming that they were the fundamental resonance.

which the FBG is inscribed. To suppress cladding mode coupling, specialty fibers were developed which possessed UV-sensitive cladding as well as core regions. When the FBG was inscribed uniformly across both photosensitive regions of the fiber-cross section where the $\mathrm{LP}_{01}$ mode had nonzero power, coupling to cladding modes was eliminated [38]. The major drawback of this technique was that these specialty fibers are costly to produce, and fiber photosensitivity also needed to be enhanced by $\mathrm{H}_{2}$-loading.

Initial FBG inscriptions with the femtosecond-IR laser and the phase mask showed that structures could be written with almost equal ease in either all-silica core or Ge-doped silica core fibers without $\mathrm{H}_{2}$-loading [12]. By implementing the order-walk effect and beam scanning, high-quality gratings were inscribed in both the core and cladding region of standard SMF-28 fiber in such a way that the $\mathrm{LP}_{01}$ mode was completely subtended by grating structure in the core and cladding (see Figure 10). This resulted in an almost complete suppression of the coupling into cladding modes as shown in Figure 11 [39]. In [39], a third-order grating with $\Lambda_{G}=1.6 \mu \mathrm{m}$ was used in order to observe the structure directly under an optical microscope. Similar FBGs written at the fundamental grating pitch of $\Lambda_{G}=0.535 \mu \mathrm{m}$ also demonstrated excellent cladding mode suppression but the structure could not be directly observed under the optical microscope as in Figure 10.

\subsection{Writing through the Fiber Jacket}

Standard UV-laser inscription processes of FBGs typically require the removal of the protective polymer coating prior to exposure as these coatings usually are highly absorbing in the UV. Coating removal and replacement are timeconsuming processes that threaten the mechanical integrity of the fiber and are therefore undesirable from a manufacturing viewpoint.

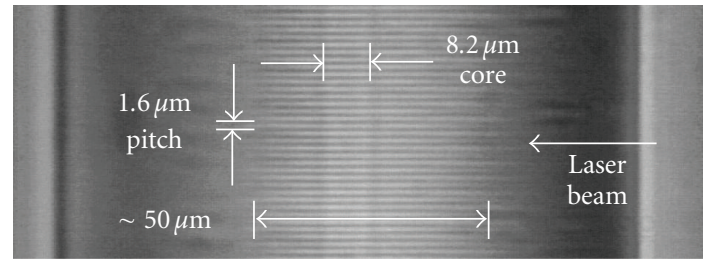

(a)

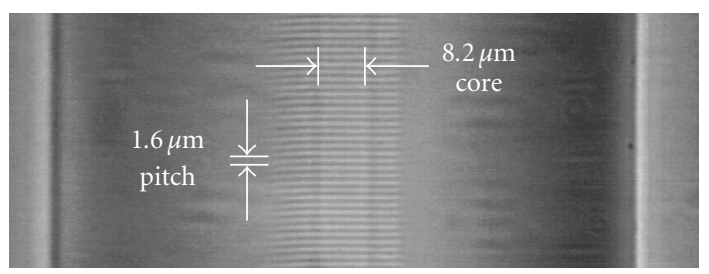

(b)

FIGURE 10: Microscope images of photoinduced index modulation in SMF-28; (see Figure 10(a)). The fiber is imaged near the core and is viewed normal to the beam axis. In Figure 10(b) the fiber is rotated $90^{\circ}$ and is viewed along the beam axis.

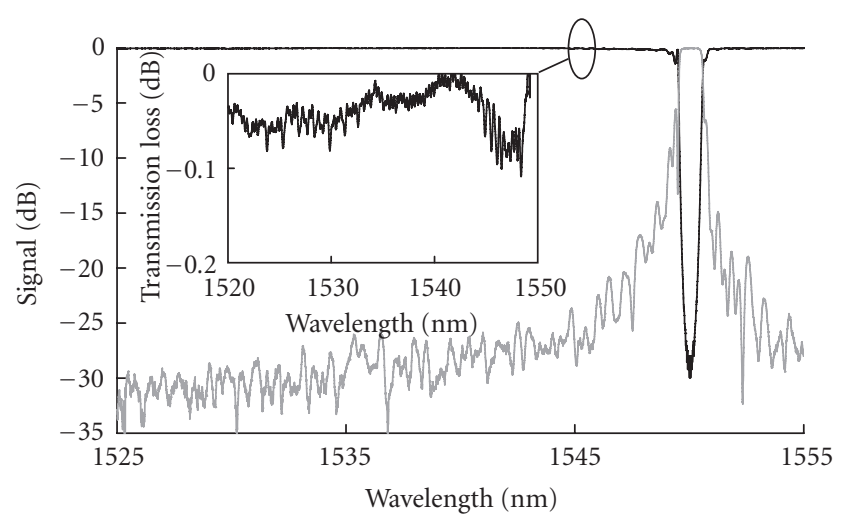

FIGURE 11: Transmission of cladding mode suppressed grating in SMF-28 shown in Figure 10. Inset figure shows the cladding mode loss expressed as difference between the transmission spectrum and 1 - $\mathrm{R}$ reflection spectrum.

Recently, FBG inscription through the standard polymer coating of a fiber was reported using femtosecond-IR radiation and the point-by-point method [40] where the tight focusing geometry was able to produce a large intensity differential between the fiber coating and the fiber core. Substantial $\Delta n$ values were induced in the core without damaging the coating $\left(\Delta n=3 \times 10^{-4}\right)$.

Using the femtosecond-IR laser and the phase mask technique, FBGs were successfully inscribed through the acrylate coating of standard $S M F-28$ fiber, when the fiber had been loaded with hydrogen [41]. A tight focusing lens was used with a focal length $f=12 \mathrm{~mm}$, but with the fiber placed at distance $d=1 \mathrm{~mm}$ behind the $1.071 \mu \mathrm{m}$ phase mask which resulted in a fundamental period grating in the fiber of $0.5355 \mu \mathrm{m}$ when irradiated with $400 \mu \mathrm{J}$ and 
125 femtoseconds pulses. The resultant FBG had a $\Delta n=$ $3.5 \times 10^{-4}$.

Under identical exposure conditions $(100 \mathrm{~Hz}, 20$ seconds cycle of $\pm 10 \mu \mathrm{m}$ beam sweep and pulse energies of $220 \mu \mathrm{J})$, grating growth as a function of time was compared for gratings written through the acrylate coating of the $\mathrm{H}_{2}$ loaded $S M F-28$ with growth rates obtained when the loaded $S M F-28$ fiber had been chemically stripped. In Figure 12(a), significant grating growth is initially observed for FBGs written through the jacket. However after $\sim 5$ minutes exposure, no further increase in $\Delta n$ was observed. For the stripped fiber, the grating growth continues even after a 90-minute exposure (see Figure 12(b)). Observation of the exposed region of the polymer jacket under the microscope revealed some damage to the jacket surface. Several femtosecond IR laser-based ablation studies of polymeric materials have shown that the threshold for induced index change and polymer ablation decreases with the number of incident pulses $[42,43]$. It is likely that the first several hundred pulses incident on the acrylate polymer coating are creating "incubation sites" that eventually reduce the transmission of the IR radiation through the jacket thus limiting the $\Delta n$ growth. Using an optical fiber pull tester (Vytran PTR-100), the breakage strength was compared to pristine $S M F-28$ fiber. For the 5 devices that were tested, the breakage strength was $4.5 \pm 0.5 \mathrm{GPa}(690 \pm 80 \mathrm{kpsi})$ as compared to $5.3 \pm 0.3 \mathrm{GPa}$ $(770 \pm 40 \mathrm{kpsi})$ for the pristine fiber.

In another set of experiments, FBGs were inscribed through the acrylate coatings of high-Ge content high NA SM1500(4.2/125) fibers from FiberCore. Using exposure conditions similar to those used with the $S M F-28$ fibers, but with pulse energies up to $440 \mu \mathrm{J} / \mathrm{pulse}$, high index modulations were induced through the jacket of this high$\mathrm{NA}$ fiber both with and without $\mathrm{H}_{2}$-loading [44]. Figure 13 displays the growth rate of the $\Delta n$ as a function of exposure for $\mathrm{H}_{2}$-loaded fiber. Index modulation values approaching $10^{-2}$ are realized with the jacket removed. As with standard SMF fiber, saturation in the induced index change through the jacket is observed. The inset image of Figure 13 displays the transmission spectra of gratings written through the acrylate jacket with and without hydrogen loading producing $\Delta n$ of $1.4 \times 10^{-3}$ and $7 \times 10^{-4}$, respectively.

For downhole monitoring applications in the oil and gas industry, FBG sensors are written into optical fibers coated with polyimide, which is thermally stable up to $300^{\circ} \mathrm{C}$. For standard UV grating inscription, the polyimide coating needs to be removed, as it is strongly absorbing in the UV. Polyimide is resistant to chemical attack. Coating removal is then a nontrivial exercise requiring immersion of the fiber in a hot sulfuric acid bath. Using the femtosecond-IR laser phase mask technique, we were able to directly inscribe gratings through the polyimide jacket of commercially available high-NA fibers from OFS-Fitel (ClearLite CL-POLY1310-21) after the fiber was $\mathrm{H}_{2}$-loaded.

For unloaded OFS fiber, the maximum FBG reflectivity achievable was $1 \%$. A reflection spectrum of the device is shown in Figure 14(a). The Bragg resonance of the FBG, $\lambda_{B}$, is $1555.24 \mathrm{~nm}$, which corresponds to an effective core mode index $n_{\text {eff }}=1.452$. Similar FBGs written in SMF-28

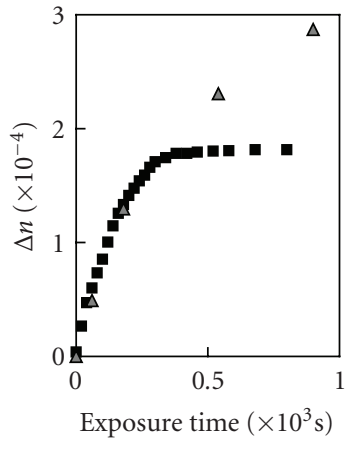

(a)

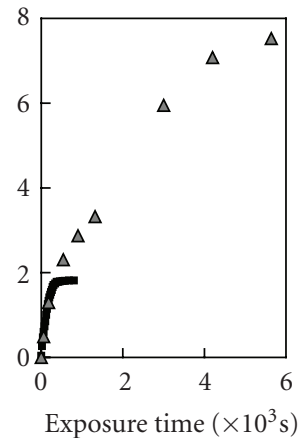

(b)
FIgURE 12: (a) Increase in $\Delta n$ as a function of exposure time for gratings written through the polymer coating with $250 \mu \mathrm{J}$ pulses and a $f=19 \mathrm{~mm}$ cylindrical lens (black squares) and gratings written on stripped SMF-28 fibre (gray triangles); (b) Long-term continued grating growth on stripped $S M F-28$ fiber.

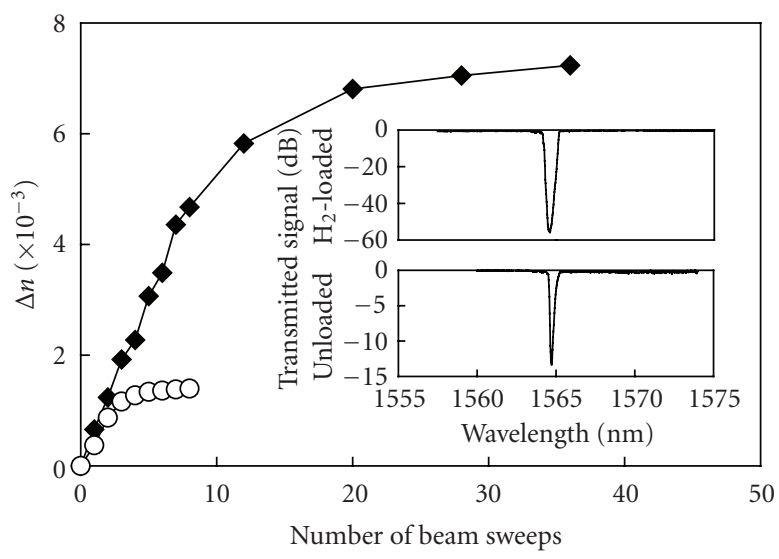

FIGURE 13: Grating growth in $\mathrm{H}_{2}$-loaded FiberCore fiber through the jacket (white circles) and with jacket removed (black diamonds). Inset image is of transmission spectra of gratings written through thejacket with (top) and without (bottom) $\mathrm{H}_{2}$-loading.

result in a $n_{\text {eff }}=1.447$, implying that there is a higher Gedopant concentration in the high-NA fiber. The transmission spectrum of an FBG written in $\mathrm{H}_{2}$-loaded OFS fiber with pulse energies of $180 \mu \mathrm{J}$ is presented in Figure 14(b). The total exposure time for the hydrogen loaded grating inscription was 5 minutes, after which no further grating growth was observed. For both the loaded and unloaded OFS fibers, the induced-index change for the gratings occurred in type I-IR regime. Using the Rouard method, the transmission spectrum of the grating was modeled assuming a Gaussian index profile $6.4 \mathrm{~mm}$ in length and a 5 -photon process. The modeled response is shown as the dotted line in Figure 14(b). From the model, the maximum index modulation in the core corresponding to the experimental data is $\Delta n=4.8 \times 10^{-4}$. For the unloaded case, the reflectivity of $1 \%$ corresponds to a $\Delta n=3 \times 10^{-5}$.

Observation of the exposed region of the polymer jacket under an optical microscope revealed some damage to the 


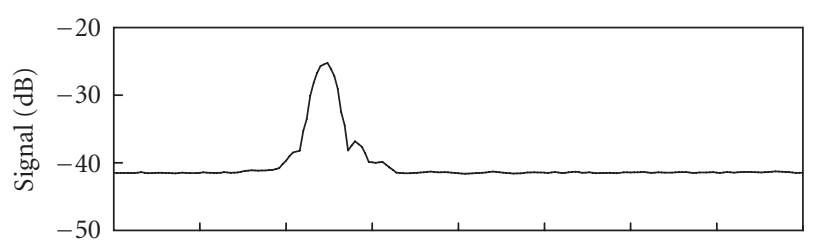

(a) Wavelength (nm)

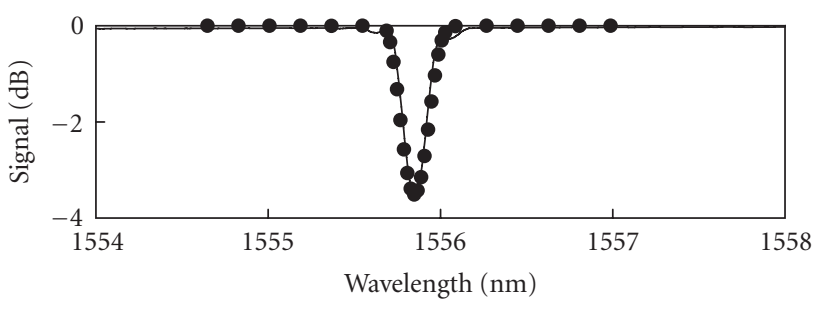

(b) Wavelength (nm)

Figure 14: (a) Reflection spectrum of an FBG written through the polyimide jacket of the OFS fiber without $\mathrm{H}_{2}$-loading; (b) transmission spectrum of an FBG written through the polyimide jacket of $\mathrm{H}_{2}$-loaded OFS fibre. Overlaid modeled spectrum shown with black dots.

jacket surface after the 5-minute exposure. FemtosecondIR laser machining studies of polyimide have shown that the ablation threshold for polyimide decreases with the number of incident pulses [43]. It is likely that the first several hundred pulses are inducing "incubation sites" in the polyimide polymer coating that eventually reduces the transmission of the IR radiation through the jacket thus limiting the $\Delta n$ growth.

To study the FBG temperature stability, isochronal annealing studies were performed on the gratings at elevated temperatures. In Figure 15, the reduction of $\Delta n$ at various elevated temperatures is shown for a grating with an initial $\Delta n$ of $3.6 \times 10^{-4}$. A portion of the $\Delta n$ is rapidly annealed out at $200^{\circ} \mathrm{C}$ but the remaining $\Delta n$ is relatively stable with increasing temperature up to $400^{\circ} \mathrm{C}$. After 16 hours at $400^{\circ} \mathrm{C}$, the grating had a $\Delta n$ of $2 \times 10^{-4}$ with no visible erasure. As the rated operating temperature of the fiber coating is $300^{\circ} \mathrm{C}$, it is likely that the grating reflectivity will remain stable within the operating temperature of the coating. When the fiber temperature was increased in $100^{\circ} \mathrm{C}$ increments and annealed for 1 hour at each temperature, grating erasure was observed with complete grating erasure occurring at $800^{\circ} \mathrm{C}$. In a separate experiment, another device was annealed at $360^{\circ} \mathrm{C}$ for 140 hours after being preannealed at $75^{\circ} \mathrm{C}$ for 36 hours in order to out-gas any remaining hydrogen (see Figure 16). After 140 hours, a $\Delta n \gg 2.2 \times 10^{-4}$ remained.

\subsection{Gratings in Active Fibers for Fiber Lasers}

Fiber lasers have become essential components for telecom and sensing applications because of their high efficiency, gain, and good beam quality. Writing Bragg gratings reflectors directly into the core of active single mode optical fibers is a convenient way to create compact, fiber compatible,

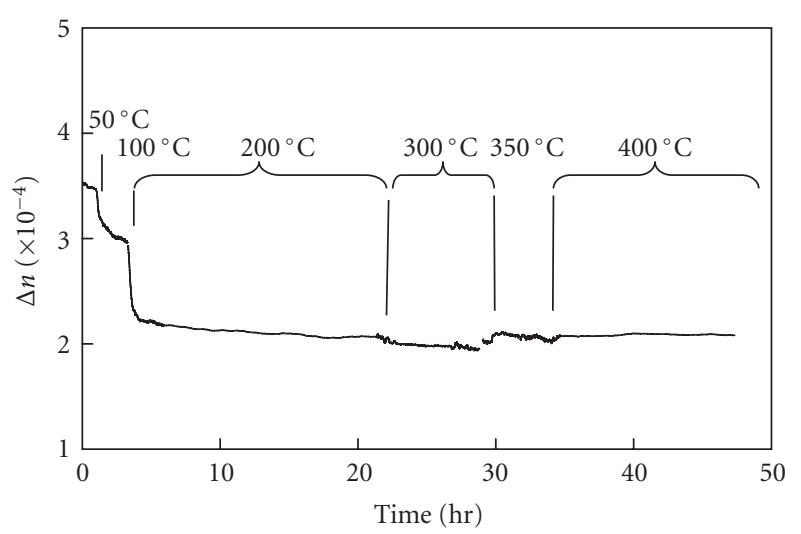

FIGURE 15: Isochronal annealing study of an FBG in the polyimide coated OFS fiber.

and low-loss laser resonator cavities. Traditional UV laser inscription of fiber Bragg gratings (FBGs) however is not appropriate for short cavity fiber lasers where high levels of Erbium-Ytterbium doping $\left(\mathrm{Er}^{3+}\right.$ and $\left.\mathrm{Yb}^{3+}\right)$ are required [45]. Germanium $(\mathrm{Ge})$ codoping of the fiber core, needed to enhance UV photosensitivity, causes ion clustering of $\mathrm{Er}^{3+}$ and $\mathrm{Yb}^{3+}$ core dopants resulting in low laser efficiency [46]. Glass substrates other than silica, for example fluoride or phosphate glass, are more desirable host materials for active dopants as higher concentrations of $\mathrm{Er}^{3+}$ and $\mathrm{Yb}^{3+}$ can be realized. Dissimilarity with silica however makes coupling of these fibers to external resonator mirrors difficult. Direct inscription of FBG resonators in these fibers would be preferable.

Recently, femtosecond-IR lasers were used to inscribe grating reflectors directly into conventional, nonphotosensitive, silica-based Er:Yb-codoped fiber by the point-by-point [47], or phase mask techniques [48] for the manufacturing of fiber lasers. These gratings successfully resulted in lasing of the fiber laser cavities. In our laboratories, short strong gratings were induced in heavily doped $\mathrm{Er}-\mathrm{Yb}$ phosphate glass fiber producing resonator cavity reflectivities above $99.99 \%$ for a $6-\mathrm{mm}$-long grating corresponding to $\Delta n>$ $1.5 \times 10^{-3}$ [15]. Femtosecond IR laser-induced gratings have also been fabricated in undoped [17] and doped fluoride fibers [49].

The impact, however, of high active doping levels on femtosecond-IR laser inscription of FBGs was unclear. In order to develop recipes for laser cavity grating inscription, investigations to determine optimal exposure conditions and active doping levels in silica-based fibers were performed [18]. High $\Delta n$ FBGs were written in commercially available fibers with different concentrations of $\mathrm{Er}^{3+}$ and/or $\mathrm{Yb}^{3+}$ in order to study the magnitude of FBG reflectivity and spectral quality as a function of active doping levels. For each fiber that was studied, the saturation in induced $\Delta n$, the spectral quality and out-of-band loss were evaluated and compared with gratings made in pure silica core single mode fibers.

Various active fibers with different levels of doping were tested. Since $\mathrm{Er}^{3+}$ and $\mathrm{Yb}^{3+}$ dopant concentration values were unavailable, it was assumed that they were 
TABLE 1: Active fibers evaluated.

\begin{tabular}{|c|c|c|c|c|c|c|}
\hline \multirow[t]{2}{*}{ Fiber } & \multirow[t]{2}{*}{ Dopant } & \multicolumn{2}{|c|}{$\operatorname{Loss}(\mathrm{dB} / \mathrm{m})$ at $\lambda \mathrm{nm}$} & \multirow{2}{*}{$\begin{array}{c}\text { Core size } \\
(\mu \mathrm{m})\end{array}$} & \multirow{2}{*}{$\begin{array}{l}\lambda_{\text {cut off }} \\
(\mathrm{nm})\end{array}$} & \multirow[t]{2}{*}{ NA } \\
\hline & & 1532 & 980 & & & \\
\hline INO EY302 & Er:Yb & 29 & 227 & $4 \times 6$ & 1145 & 0.14 \\
\hline INO EY305 & Er:Yb & 22 & 477 & 6 & 1200 & 0.18 \\
\hline INO EY550 & Er:Yb & 11.2 & 593 & $2.5 \times 9$ & 900 & 0.17 \\
\hline Coractive EY18-06 & Er:Yb & 58 & 400 & 6 & na & 0.18 \\
\hline INO Yb214 & $\mathrm{Y}$ & 0 & 612 & 6.2 & 900 & 0.12 \\
\hline INO Er406 & Er & 37 & 18 & 3.4 & 860 & 0.2 \\
\hline Corguide & Er & 25 & 10 & 6 & na & na \\
\hline Brown University & Er & 12 & 4 & 8 & na & na \\
\hline
\end{tabular}

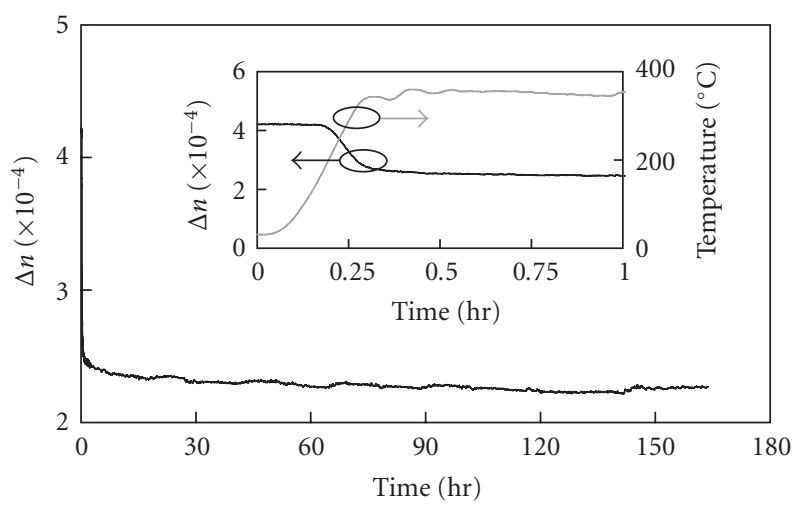

FIGURE 16: Long-term annealing study at $360^{\circ} \mathrm{C}$ of an FBG in OFS fiber. Inset is an expanded view of the initial annealing with $\Delta n$ and temperature denoted by black and gray traces, respectively.

proportional to the losses given at telecommunication and pump wavelengths, respectively, as specified in Table 1 . The FBGs were written using a first-order $1.07 \mu \mathrm{m}$ pitched phase mask positioned $1 \mathrm{~mm}$ away from the fibers. All exposures were made using $1.2 \mathrm{~mJ}$ pulses of IR radiation with laser repetition rates set at $100 \mathrm{~Hz}$.

Grating growth was very rapid, producing Bragg resonances of $-40 \mathrm{~dB}$ after only a few seconds of exposure. All FBGs made in the doped active fibers listed in Table 1 share two characteristics: the initial FBG growth rate exceeds that of pure silica core fibers (see Figure 17(a)) and the growth of the $\Delta n$ in all the FBGs made in active fibers saturate well below the pure silica core fiber levels of $4 \times 10^{-3}$. For FBGs in active fiber, the saturated $\Delta n$ was estimated to be 2 to $3.5 \times 10^{-3}$ (see Figure 17(b)).

The plot of the saturated $\Delta n$ versus the absorption at $1532 \mathrm{~nm}$ and $980 \mathrm{~nm}$ for the gratings was highly scattered (see Figure 18). Error bars denote the average values of three gratings written in the fiber. A weak negative correlation between saturated $\Delta n$ 's and increasing $\mathrm{Er}$ and $\mathrm{Yb}$ content of the active fibers was observed. The large scatter of the saturated $\Delta n$ 's may be due to the different glass composition between the fibers that were studied. With all the doped fibers tested, spectral quality and out-of-band insertion losses were

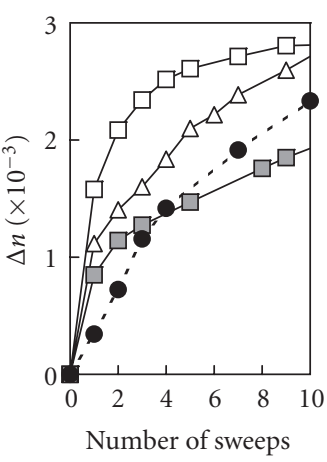

(a)

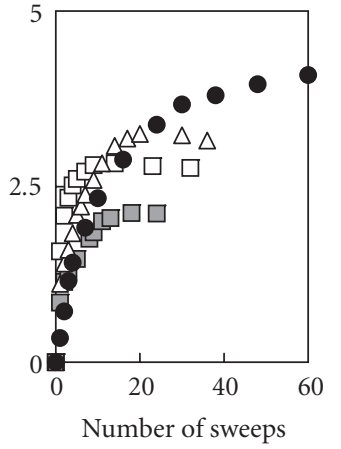

(b)
FIGURE 17: (a) Initial growth and saturated growth (b) of $\Delta n$ versus IR exposures for different active fibers and for pure silica core fiber (black dots). White triangles denote EY302, white squares denote EY305, and gray squares denote Er406.

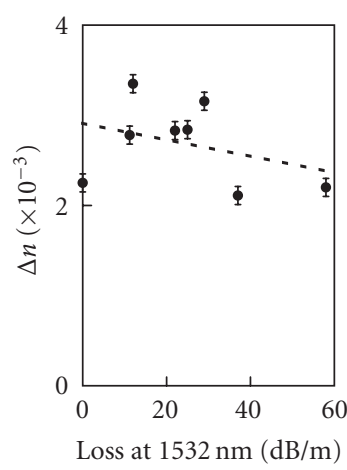

(a)

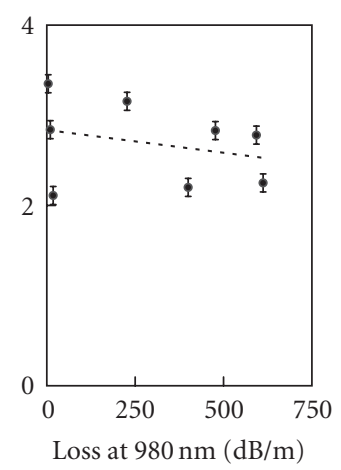

(b)
FIGURE 18: Saturation levels of the active fibers $\Delta n$ 's as a function of (a) loss at $1550 \mathrm{~nm}$ and (b) loss at $980 \mathrm{~nm}$. Error bars denote the averaging of the values obtained from the three sequential exposures of the fiber. Dotted lines denote the linear regression through the data points.

similar to the cladding mode suppressed gratings in standard fiber presented in Figure 11. 


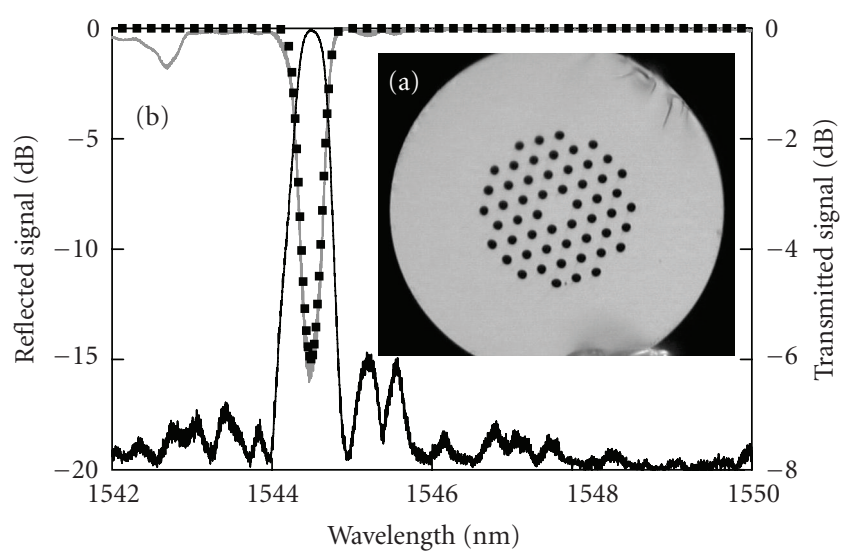

Figure 19: (a) Cross section of ESM-12-01 fiber. (b) Transmission (gray), reflection (black), and modeled (dotted) spectra of a grating written in ESM-12-01 fiber with $1200 \mu \mathrm{J}$ pulse energy $200 \mathrm{~Hz}$. Total exposure time 5 minutes.

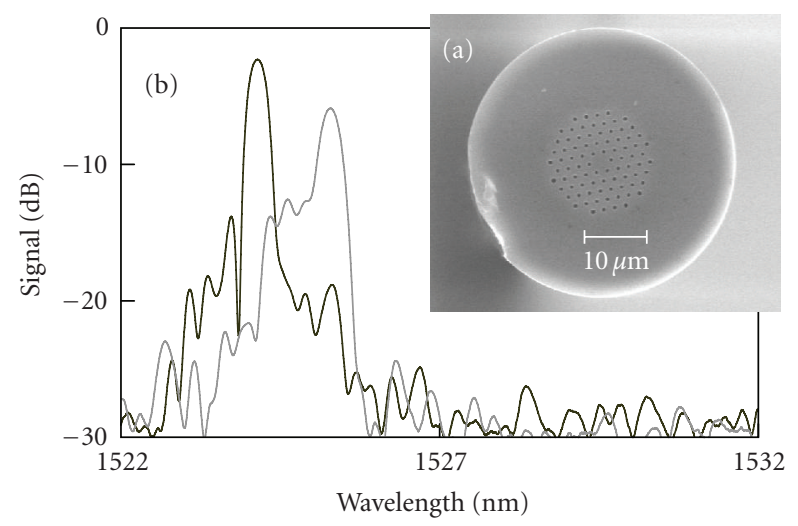

Figure 20: (a) Scanning electron microscope image of $45 \mu \mathrm{m}$ diameter LMA-15 taper, (b) reflection spectra of grating in $45 \mu \mathrm{m}$ LMA-15 fiber taper for two orthogonal polarizations.

\subsection{Gratings in Photonic Crystal Fibers}

Inscription of FBG structures in photonic crystal fibers (PCFs) enhances the flexibility of the PCF for telecommunication as well as microfluidic sensing related applications. Direct inscription of FBGs in pure silica PCF has been achieved using ArF excimer laser irradiation as well as femtosecond UV irradiation producing $\Delta n \sim 2 \times 10^{-4}$ after a 3.8-hour write time and $\sim 1 \times 10^{-4}$ after a one hour exposure time $[9,50]$.

Using the femtosecond-IR laser-phase mask approach, FBGs were written in pure silica PCF and fiber tapers [20]. Scattering of the writing beam by the PCF cladding holes can be reduced by selecting fiber geometries with smaller number of cladding holes between the fiber surface and the core. With such a PCF, for example the ESM-12-01 fiber from Blaze Photonics/Crystal Fibre, which has 3 rows of air holes surrounding the silica core, a $\Delta n$ value of $\sim 4 \times 10^{-4}$ was obtained after a 5-minute exposure with a $\Lambda_{m}=3.12 \mu \mathrm{m}$ mask, tight focusing $(f=30 \mathrm{~mm})$, and high pulse energy $(1200 \mu \mathrm{J} /$ pulse at $200 \mathrm{~Hz})$. The spectral response of the grating in the ESM-12-01 fiber is shown in Figure 19. For other PCF with larger number of air holes, scattering of the incident IR light did not result in any grating fabrication in the core, even at pulse energies that resulted in damage to the fiber. By tapering such PCF in a $\mathrm{H}_{2} / \mathrm{O}_{2}$ flame, the fiber diameter could be reduced causing the bandgap generated by the cladding holes as seen by the transversal IR beam to shift to a wavelength that was not resonant with the IR source. The resultant narrower hole diameter and spacing in the tapered PCF allowed partial transmission of the IR radiation into the core resulting in FBGs in the tapered regions of the PCF with $\Delta n \sim 3 \times 10^{-4}$ after an 80second exposure. Alternatively, the holes of the PCF could be collapsed altogether. Gratings inscribed in the collapsed region across the entire cross section of the taper diameter produced multimode transmission/reflection responses.

The tapering of the PCF by the $\mathrm{H}_{2} / \mathrm{O}_{2}$ flame such that the air holes was not collapsed but still produced a high level of birefringence in the tapered region [51]. LMA-15 PCF from Crystal Fibre A/S was tapered from its initial $230 \mu \mathrm{m}$ outer diameter to a taper waist diameter of $45 \mu \mathrm{m}$ and $10 \mathrm{~mm}$ length. The fiber taper still guided the fundamental mode without any loss. By inscribing a weak grating in the PCF core of the taper region, the birefringence of the PCF taper was quantified. In Figure 20, the grating was probed with polarized light from a tunable laser source and the spectral characteristics of the gratings made in the tapered PCF show a strong dependence on the signal polarization. When the polarization of the signal was rotated using an inline fiber polarizer, it was possible to switch the main reflection spectrum from the Bragg resonance wavelength at $1525.2 \mathrm{~nm}$ to a lower wavelength of $1524.1 \mathrm{~nm}$. For a birefringent Bragg grating, the wavelength shift between the two orthogonal polarizations $\Delta \lambda_{B}$ will be related to the difference between the $n_{\text {eff }}$ of the two modes $\Delta n_{\text {eff }}$ by: $\Delta \lambda_{B} / \lambda_{B}=\Delta n_{\text {eff }} / n_{\text {eff }}$ which results in a $\Delta n_{\text {eff }}$ or birefringence value of $1.05 \times 10^{-3}$.

\subsection{Induction of High Birefringence}

For some applications, a given amount of birefringence in an optical device or system is desirable, among the most important being the single polarization fiber lasers [52] and Bragg grating sensing [53]. Typically, highly birefringent fibers of complex design are used in these specialized applications. The birefringence, however, could be introduced locally by processing the optical substrate with high-energy laser pulses. Small amounts of birefringence $\left(4 \times 10^{-5}\right)$ can be created with UV exposure of Ge-doped fibers [54] by controlling the polarization of the UV-writing source so that it was normal to the optical fiber axis.

Similarly, type I-IR FBGs induced with a phase mask uniformly about the core have been shown to produce similar levels of birefringence when the polarization of the IR beam was also polarized normal to the fiber axis $\left(\sim 10^{-5}\right)$ [55]. In the type II-IR regime, FBGs written uniformly about the fiber core produced levels of birefringence approaching $10^{-4}$, an order of magnitude higher than the birefringence 
resulting from the type I-IR exposure. Although the type II-IR gratings potentially produce enough birefringence for most applications, one draw back of these structures is that there is typically increased insertion loss for gratings with stronger $\Delta n$ 's. For applications such as short cavity single polarization lasers, the increased loss associated with the birefringent laser resonator mirror would reduce the laser performance.

In our laboratories, we have shown that if a type II-IR grating structure is made in the cladding close to but not overlapping the core, such that there is no retroreflectance from the structure, a large amount of birefringence is locally induced in the core region without generating excess loss [56]. Birefringence values as high as $\sim 8 \times 10^{-4}$ were obtained in the core of SMF-28 fiber when type II-IR grating structures were inscribed in the cladding of the fiber on both sides of the core in close proximity to the core/cladding interface. The splitting of the reflection spectrum of a weak probe grating written in the core is shown in Figure 21 along with a schematic of the exposure which produced the high birefringence result and a photomicrograph of the grating induced in the cladding. The birefringence is likely due to induced stress rather than direct asymmetric refractive index modification.

When a strong type I-IR grating ( $\sim 95 \%$ reflectivity) is present in the core, the type II-IR cladding exposures resulted not only in increased birefringence of the effective core mode, as measured by the induced polarization dependent wavelength shift, but also resulted in a large grating strength difference between orthogonal polarization axes $(\Delta \operatorname{Tr})$ for the Bragg resonance. As can be seen in Figure 22, the ordinary polarization corresponds to a $15 \mathrm{~dB}$ strong grating while the extraordinary polarization results in a secondary resonance of $5 \mathrm{~dB}$ located $540 \mathrm{pm}$ away from the main resonance $\left(\sim 5 \times 10^{-4}\right.$ birefringence) resulting in $\Delta \operatorname{Tr}=$ $10 \mathrm{~dB}$. The difference in the $\Delta n$ with polarization based on the evaluation of the refractive index modulation of the two gratings resulted in a value of $\sim 2 \times 10^{-4}$ assuming the approximation of a fundamental (first-order) grating. Since the spectrum of the original grating measured before the cladding exposure did not display a large $\Delta \operatorname{Tr}$ at the Bragg resonance, it can be assumed that the initial $\Delta n$ was similar for the two principle states of polarization and the change in $\Delta \operatorname{Tr}$ was a result of the cladding exposure.

\subsection{High-Temperature Stable Sensor Gratings}

A very important application of FBG technology is its usage as sensing elements. FBGs are capable of monitoring both temperature and strain through variations in the spectral response of the grating, which can be directly correlated to strain and temperature imposed on the grating structure [57]. The applicability of traditional UV laser-induced FBGs is restricted to lower temperatures $\left(<600^{\circ} \mathrm{C}\right)$ because of the instability of the UV generated grating structure at higher temperatures. High-temperature grating stability is especially important for sensing applications such as monitoring furnaces, combustion situations, and so forth.

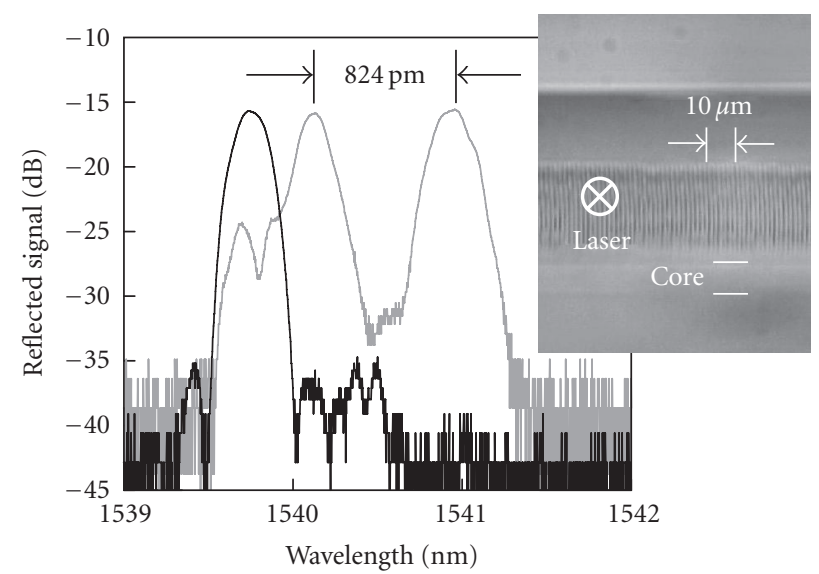

(a)

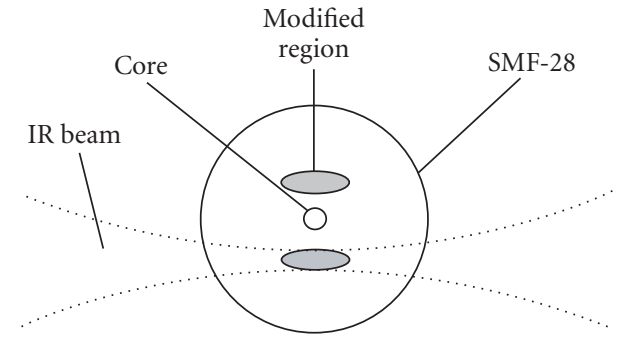

(b)

FIGURE 21: The spectra of the probe grating before and after the cladding exposure are denoted by black and grey traces, respectively. Inset photomicrograph shows a type II-IR exposure in $S M F-28$ fiber in the cladding proximate to the core. Image taken along the exposure beam axis (b) shows a schematic representation of the exposure conditions that produced the high birefringent spectral result.

Femtosecond IR laser-induced gratings in standard SMF28 and all silica core single mode fiber have displayed high long-term thermal stability at $1000^{\circ} \mathrm{C}$ when the $\mathrm{FBGs}$ were inscribed in the type II-IR regime [12]. Above $1000^{\circ} \mathrm{C}$, the silica substrate itself is susceptible to structural transformations that limit the functionality of the fiber [58]. For temperatures $>1000^{\circ} \mathrm{C}$, fiber substrates other than silica need to be considered. The most successful optical fiber used for high-temperature sensor applications is the single crystal sapphire fiber that has a glass transition temperature $t_{g}$ of $\sim 2030^{\circ} \mathrm{C}$. Unlike conventional silica-based single mode optical fibers, sapphire fibers are made in the form of rods absent a cladding layer. With fiber diameters commercially available, beam propagation within the fiber is highly multimode at the $1550 \mathrm{~nm}$ telecommunication wavelengths. Present sapphire fiber sensors are mostly based on FabryPerot structures within the fiber producing a broadband interferometric signal that varies with temperature [59].

Femtosecond IR-laser induced gratings in sapphire fiber show no degradation of the grating strength at high temperatures up to $1500^{\circ} \mathrm{C}[15,16]$. The sapphire FBGs (SFBG) have definite advantages over other sapphire fiber sensors 


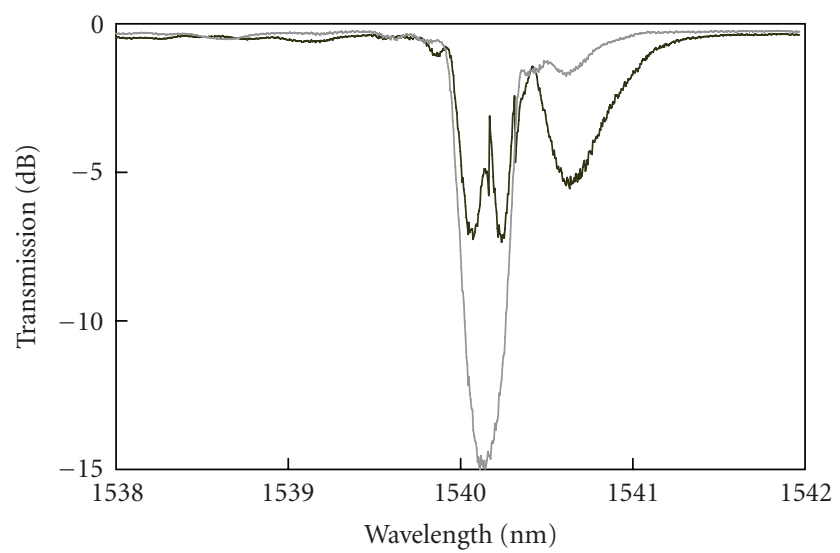

FIGURE 22: Large birefringence of the refractive-index modulation induced by a symmetric cladding exposure of a strong type I-IR core grating. The two orthogonal polarization states are denoted by the gray and black traces, respectively.

that rely on Fabry-Perot etalons at the fiber tip. Unlike FabryPerot sapphire sensors, SFBG sensors with their discrete resonant wavelength could potentially be used as distributed optical sensor arrays up to $2000^{\circ} \mathrm{C}$. The SFBGs that are inscribed in sapphire fiber using femtosecond-IR lasers and a phase mask are naturally multimode devices. The multimode reflection spectrum observed with the retroreflective SFBGs is characterized by a large bandwidth having a complicated structure that consists of a superposition of different modes reflected by the grating. Although the SFBG sensor can be used in the multimode regime, propagation of a single mode is preferable due to stability and the detection advantages that a narrowband single mode reflection response offers. To produce a single mode response, the multimode SFBGs were probed using tapered single mode fibers. Single and loworder mode reflection/transmission responses are produced when the mode field of the launched single mode is matched with the fundamental mode supported by the sapphire fiber. For a $150 \mu \mathrm{m}$ diameter sapphire fiber, this would correspond to an $\mathrm{LP}_{01}$ mode field $\sim 150 \mu \mathrm{m}$ in diameter. When single mode optical fiber is tapered such that the normalized frequency or $V$ number of the core is $<1$, the fundamental $\mathrm{LP}_{01}$ mode is no longer core guided but is guided by the cladding-air interface resulting in a mode field with the same diameter as the tapered fiber. Matching taper and sapphire fiber outer diameters may excite only a single mode of the sapphire fiber. The evanescent tapers were made from fibers with different cutoff wavelengths $\lambda_{\text {cut }}$.

Although, theoretically less efficient for mode matching, the expanded fundamental mode of a single mode fiber can also be used to reduce the number of modes excited by the probing signal. As in the case of tapers, the single mode probe signal from a fiber collimator can be launched from a single mode $1550 \mathrm{~nm}$ optical circulator into the SFBG, and the reflected signal can be detected at the third port of the circulator instead of using the less efficient fiber couplers. A configuration made of an input single mode tapered fiber and multimode silica fiber used for output coupling

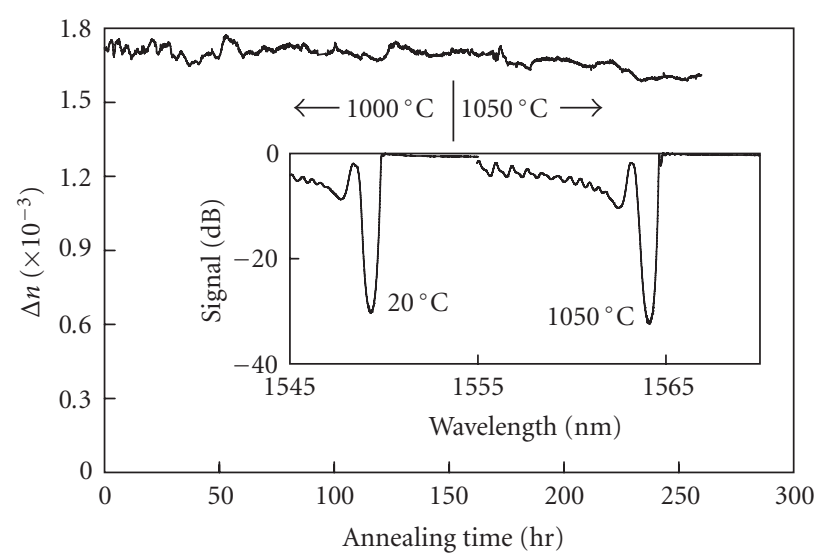

Figure 23: Long-term annealing at 1000 and $1050^{\circ} \mathrm{C}$ of a femtosecond IR FBG. Inset displays the initial room temperature transmission spectrum and the final spectrum at $1050^{\circ} \mathrm{C}$ for 100 hours.

was also tested and has delivered a filtered multimode transmission spectrum. The tapered coupling improved the spectral resolution of the sapphire FBG as compared to its multimode responses. Such improvements facilitate the utilization of the sapphire FBG as a high-temperature sensor.

\subsubsection{Thermally Stable FBGs in $\mathrm{SIO}_{2}$ Based Fibers}

FBGs written with ultrafast IR radiation exhibit different thermal stability characteristics depending upon the pulse duration and energy. Above a low-intensity threshold for grating formation (type I-IR) [23], $\Delta n$ values with similar thermal stability to UV-induced gratings can be generated as was shown in Figure 3. At higher power levels that are consistent with white light generation in the fibers, thermally ultra-stable defects/damage sites could be produced (type IIIR).

A long-term study of type II-IR grating stability was performed by inscribing large $\Delta n$ type II-IR FBGs in SMF28 fiber which were then heated to $1000^{\circ} \mathrm{C}$ in steps of $100^{\circ} \mathrm{C}$ for 1 hour at each temperature [58]. Once $1000^{\circ} \mathrm{C}$ was reached, the grating was then kept at constant temperature for 150, hours and the grating reflectivity and resonant wavelength were monitored (see Figure 23). There was no noticeable degradation of the increased grating strength for the duration of the test and the grating maintained $\Delta n=$ $1.7 \times 10^{-3}$. The initial increase in the femtoseconds grating $\Delta n$, shown in Figure 3 previously, is likely a result of the two kinds of index change being written simultaneously. The peaks of the complex interference pattern are sufficiently intense to ultimately ionize the glass in the fiber producing an index change that is durable with temperature. In the valleys of the interference pattern, the intensity is below the type II-IR threshold, however some type I-IR index change is generated. As the device is annealed, the permanent type II-IR index change remains while the annealable type IIR index change is erased resulting in a higher $\Delta n$. The 


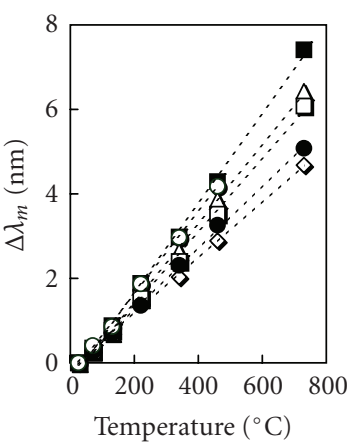

(a)

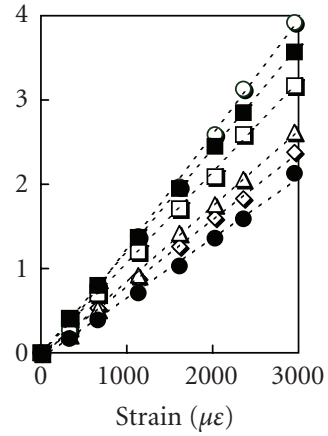

(b)
FIgURE 24: (a) Bragg-order wavelength variation as a function of temperature for the grating shown in Figure 9, (b) Braggorder wavelength variation as a function of strain. Orders $m=$ $7,8,9,10,11$, and 12 are denoted by white circles, black squares, white squares, triangles, diamonds, and black circles, respectively.

temperature of the femtoseconds grating was subsequently increased and kept at $1050^{\circ} \mathrm{C}$ for 100 hours (see Figure 23). The $\Delta n$ of the femtoseconds grating decreased slightly from $1.7 \times 10^{-3}$ to $1.6 \times 10^{-3}$ during 100 hours at $1050^{\circ} \mathrm{C}$. A drift of the Bragg resonance to longer wavelengths of $0.2 \mathrm{~nm}$ was detected at the end of experiment. Spectra taken initially at room temperature and after 100 hours at $1050^{\circ} \mathrm{C}$ are shown in inset of Figure 23.

\subsubsection{High-Temperature Multiparameter Sensor in $\mathrm{SIO}_{2}$}

As presented in Section 4.6 above, the type II-IR FBG written in Corning 980 Flexcore fiber with a cutoff wavelength of $980 \mathrm{~nm}$ and the $4.28 \mu \mathrm{m}$ pitched phase mask has many Fourier components in its spectral response that produces Bragg resonances at various wavelengths (see Figure 9). The large number of spectral resonances generated by a single grating structure is available to be used for multiparameter sensing. Aside from strain and temperature, other parameters like bending, pressure, and so forth could be simultaneously measured using only one grating. The availability of supplementary spectral characteristics could also be used to improve the accuracy of the strain-temperature discrimination.

Wavelength shifts of the various diffracted orders as a function of the temperature in the absence of strain are shown in Figure 24(a). The temperature was changed from $\sim 20^{\circ} \mathrm{C}$ to $\sim 800^{\circ} \mathrm{C}$. The room temperature $1550 \mathrm{~nm}$ Bragg resonance wavelength was monitored in order to confirm that the thermal equilibrium was reached after $\sim 30$ minutes at each temperature. After the thermal equilibrium was reached, all the resonances of the Bragg grating were measured at constant temperature. At room temperature, the gratings were subjected to controlled stress in a specially designed jig. The strain was applied incrementally from 0 to $3000 \mu \varepsilon$. After each strain increment, the wavelength shift was monitored in the $1550 \mathrm{~nm}$ range until the measurement confirmed that the Bragg wavelength was stable, as measured by an optical spectrum analyzer. The Bragg wavelength of each diffraction order was then evaluated by scanning a $10 \mathrm{~nm}$ spectral range around each high-order resonance. Wavelength shifts of the various diffracted orders as a function of strain are shown in Figure 24(b).

Under the application of strain and temperature and of another arbitrary parameter $P$, the grating spectrum of order $m$ will undergo a wavelength shift:

$$
\Delta \lambda_{m}(\varepsilon, T, P)=K_{\varepsilon m} \Delta \varepsilon+K_{T m} \Delta T+K_{P m} \Delta P,
$$

where $\Delta \lambda_{m}$ are the wavelength shifts when the ambient parameters; stress $(\varepsilon)$, temperature $(T)$, and a generic general parameter $(P)$ are changing by the amount of $\Delta \varepsilon, \Delta T$, and $\Delta P$. Considering temperature and strain, the variations in $\Delta \lambda_{m}$ differ from order to order as shown in Figures 24(a) and 24(b). Assuming that the resonance shifts are linear and independent of each other, the slopes or $K$ values of the $\Delta \lambda_{m}$ versus $T$ or $\varepsilon$ curves can be used to setup a $2 \times 2 \mathbf{K}$ matrix which will allow for the discrimination between temperature and strain using a single Bragg grating [60]. By employing more than two resonances, the problem becomes one that is over defined, potentially resulting in reduced measurement error. Alternatively, other parameters in addition to temperature and strain could also be monitored.

\subsubsection{High-Temperature Stable FBGs in Sapphire Fiber: Multimode Response}

FBGs were fabricated in crystalline sapphire fiber with diameters of $60,100,120,150$, and $180 \mu \mathrm{m}$ by exposing the fibers to the 125 femtoseconds pulsed-IR radiation. The tests were made using the $4.28 \mu \mathrm{m}$ period phase mask that resulted in a 5 th-order Bragg resonance in the sapphire fiber at $1490 \mathrm{~nm}$. Alternatively, a $1.747 \mu \mathrm{m}$ period phase mask was used that gives a second-order Bragg resonance in sapphire fiber at $1523 \mathrm{~nm}$. The first phase-mask, although producing lower reflectivity high-order resonances, has the advantage of producing grating structures in sapphire fiber that are easily observed under an optical microscope. The shorter pitched phase mask was specially designed to generate a low-order resonance in the sapphire fiber in the telecom wavelength range. In this way, the grating growth could be conveniently monitored with an erbium white light source and a spectrum analyzer.

The sapphire FBGs (SFBGs) were fabricated using the setup shown in Figure 1. The femtosecond-IR pulses were focused with a 25-mm-focal length lens through the phase mask and onto the sapphire fiber. Since the sapphire fibers have a hexagonal cross-section, they were placed in a rotation jig that would allow the fiber to be turned along its axis in order to optimize the exposure. The femtosecond-IR beam was focused inside the fiber and then scanned normal to the fiber using a precision vertical stage in order to expose the whole cross section of the sapphire fiber to the laser beam. As the sapphire fiber is essentially a sapphire rod absent a cladding layer, the spectral response from an inscribed grating is highly multimode. To measure the multimode 


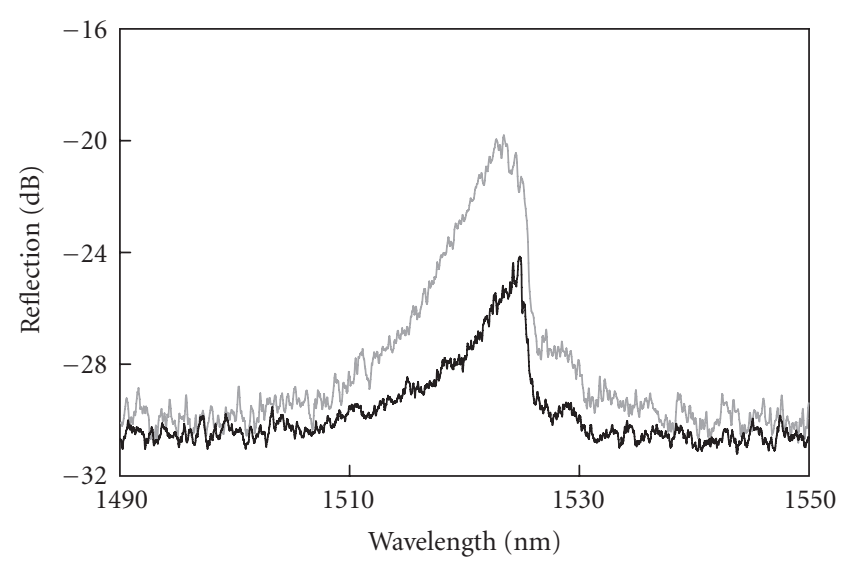

FIGURE 25: Multimode reflection spectra of the gratings written in the $150 \mu \mathrm{m}$ core diameter sapphire fiber measured from the short side (gray line) and from the long side (black line) of a $120 \mathrm{~cm}$-long sapphire fiber.

response from the SFBG, an S-C band tunable laser was passed through a 3-dB-multimode coupler made in-house with $105 / 125 \mu \mathrm{m}$ core/clad silica fiber. The output port of the coupler was mounted on 5-axis precision stages and butt-coupled to the sapphire fiber with index matching oil having a refractive index of 1.46 . The reflected response was measured using a fast detector.

SFBGs were fabricated in $150 \mu \mathrm{m}$ diameter fibers with both the 4.28 and $1.747 \mu \mathrm{m}$ phase masks producing maximum reflectivities of $8 \%$. There is a limitation on the maximum reflectivity of a multimode fiber grating due to the large number of modes that the fiber will support. In $105 \mu \mathrm{m}$ core multimode silica fiber, using standard writing conditions, the maximum reflectivity obtained using a firstorder phase mask was $30 \%$, which corresponded to a $\Delta n \sim 3 \times 10^{-3}$. This reflectivity was not reached in gratings made in sapphire with similar diameters of $100 \mu \mathrm{m}$ or even in the case of smaller diameters of 70 and $60 \mu \mathrm{m}$. It is possible that the lower reflectivity obtained in sapphire fibers as compared to silica is the result of a lower saturation level of the $\Delta n$ that can be induced in sapphire fiber using the method of ultrafast radiation and the phase mask.

The magnitude of the SFBG reflectivity also depends on the position of the grating along the sapphire fiber length. The multimode response of an SFBG written in a $120-\mathrm{cm}$ long sapphire fiber with $150 \mu \mathrm{m}$ core at $\sim 20 \mathrm{~cm}$ from one end of the fiber is presented in the Figure 25. In the figure, the multimode reflection spectrum measured from the short end is presented together with the spectral response of the same grating measured from the long side of the sapphire fiber. There is systematic $4-6 \mathrm{~dB}$ difference in the strength of the reflected signal when it propagates through 1 meter of the sapphire fiber. This loss is likely related to the propagation of the mode as well as mode conversion within the fiber. In addition to the reflectivity, the bandwidth of the grating response is also reduced which implies that higher-order modes are lost as the signal propagates along the length of the fiber.

\subsubsection{High-Temperature Stable FBGs in Sapphire Fiber: Single Mode Response}

Multimode responses from FBG sensors are not as desirable as single mode responses since the spectral bandwidth is broad and the dynamic range of the reflection signal is low. In order to achieve a quasi-single mode response from the SFBG, it was necessary to investigate other approaches to probe the grating aside from the multimode coupler. The maximization of single mode coupling to the multimode sapphire fiber can be achieved by the generation of a large mode field diameter (MFD) single mode by for example, a fiber taper [16].

For single mode fibers, the normalized frequency or $V$ number is given by

$$
V=\frac{2 \pi r}{\lambda} \sqrt{\left(n_{\mathrm{co}}^{2}-n_{\mathrm{cl}}^{2}\right)}
$$

where $r$ is the core radius, $\lambda$ is the wavelength, and $n_{i}$ is the refractive index of the core or cladding. For single mode operation, $V \leq 2.405$. When a fiber is tapered, the ratio of cladding/core radii remains constant however $V$ decreases. The transition of the fundamental $\mathrm{LP}_{01}$ mode from a corecladding guided into a cladding-air guided mode occurs when [61]

$$
V<V_{\mathrm{cc}} \cong \sqrt{\frac{2}{\ln S}}\left\{1+\frac{0.26}{\ln S}\right\}^{-1 / 2},
$$

where $S$ is the ratio of the cladding to core diameters.

Once condition (5) is met, the fundamental mode expands into the cladding resulting in a large area mode that can be better coupled into the sapphire fiber. As the expanded mode field is limited by the outer diameter of the taper, maximization of the single mode coupling occurs when the difference between the taper and the sapphire fiber diameters is minimized. Fiber tapers were fabricated using Corning Puremode HI 780 single mode fiber with an outer diameter $\phi=125 \mu \mathrm{m}$ and a cutoff wavelength $\lambda_{\text {cut }}<720 \mathrm{~nm}$ using a swept hydrogen flame while the fiber was pulled on precision translation stages. When pulled to taper diameters $\leq 70 \mu \mathrm{m}$, the fundamental mode was completely guided by the cladding air interface as shown schematically in Figure 26. Commercially available $50 \mu \mathrm{m}$ and $60 \mu \mathrm{m}$ diameter fiber collimators were also used for testing.

In order to obtain a single mode response from the sapphire grating, a probe signal from the swept tunable laser system was passed through a 1550-nm-range optical circulator and into the tapered fiber/fiber collimator. The output of the collimator and the tapers were mounted on 5axis precision stages and butt-coupled to the sapphire fiber with index matching oil having a refractive index of 1.46.

The same SFBG that produced the multimode response shown in Figure 25 was also probed with a $60 \mu \mathrm{m}$ diameter taper (see Figure 27). The reflection spectrum measured with the $60 \mu \mathrm{m}$ taper has a $3 \mathrm{~dB}$ bandwidth of $0.33 \mathrm{~nm}$ similar to that of an FBG in single mode fiber, which is compared to the multimode spectrum that has a $3 \mathrm{~dB}$ bandwidth of $6 \mathrm{~nm}$. 


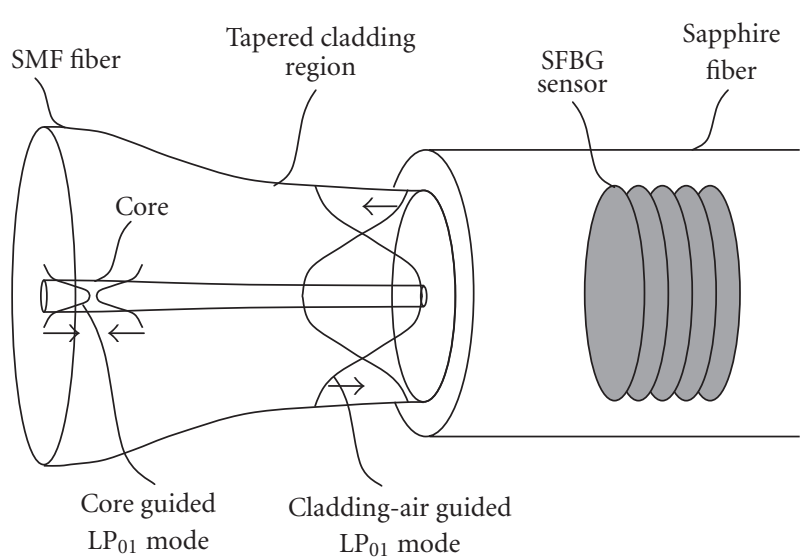

FIGURE 26: Schematic of the $\mathrm{LP}_{01}$ mode field expansion resulting from fiber tapering and coupling of the taper into the sapphire fiber.

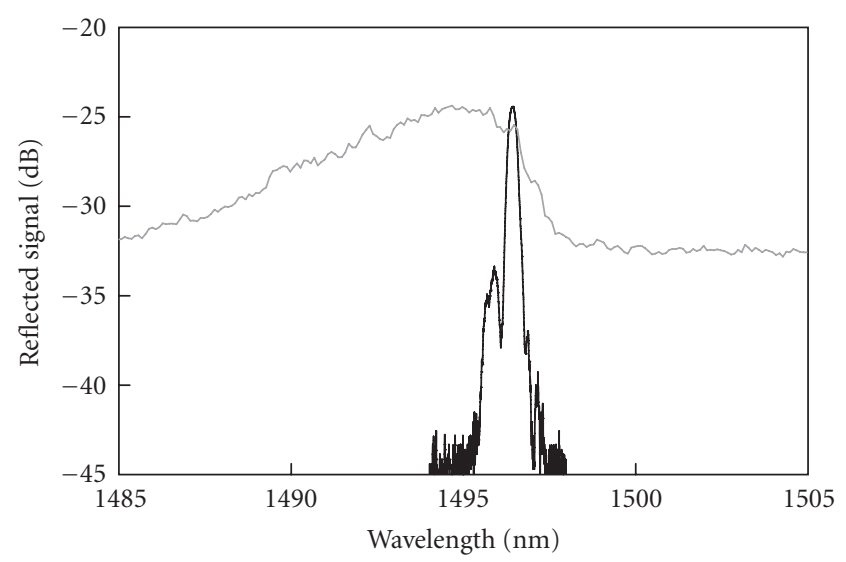

FIGURE 27: Reflection spectra of SFBG with a $100-\mu \mathrm{m}$-silica core multimode fibre (dotted line) and a 60 - $\mu$ m-taper (black line) as the input couplers.

Compared to the multimode response, there is also a $10 \mathrm{~dB}$ improvement in the signal-to-noise ratio of the $60 \mu \mathrm{m}$ taper response. The loss at the taper/sapphire splice is $4.5 \mathrm{~dB}$ per pass. This loss may be due to the mismatch of excited modes generated by and collected by the tapers.

To measure the response of the SFBG to temperature, the portion of the sapphire fiber that contained the Bragg grating was inserted into a high-temperature ceramic microfurnace (NTT model CMH-7022). In order to position the Bragg grating within the microfurnace, an He-Ne laser was connected to the input port of the multimode coupler. The red light scattered by the Bragg grating was then used to position the grating accurately within the center of the furnace where the temperature distribution was at its maximum. The microfurnace temperature was monitored at its center using a platinum-rhodium thermocouple with a measurement range up $1700^{\circ} \mathrm{C}$. A gradient with a $20 \%$ decrease in the temperature from the center to edge of the 20$\mathrm{mm}$-long microfurnace was observed. The wavelength shift of the SFBG as a function of temperature for both the single

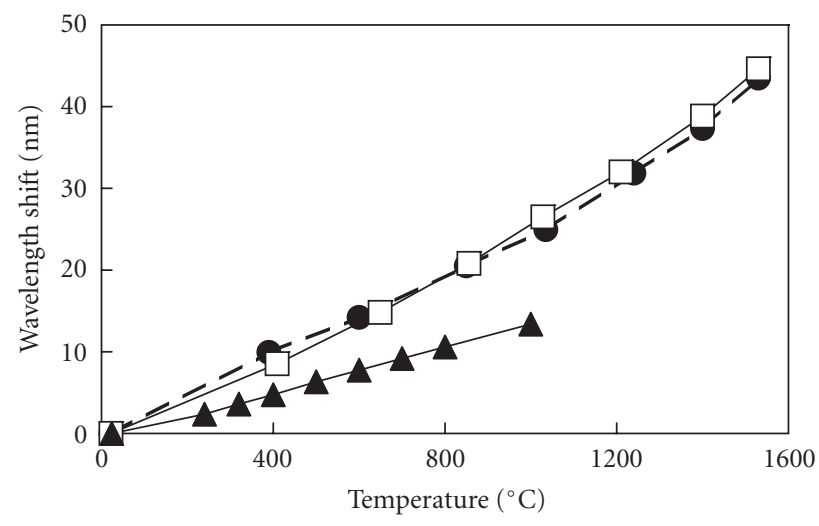

FIGURE 28: Variation of single mode (white squares) and the multimode SFBG wavelength response (black circles,) with temperature. Wavelength variation of the Bragg resonance of a type II-IR FBG written in SMF-28 fiber is also shown (black triangle).

and multimode spectral responses is presented in Figure 28. Both the single and multimode responses are similar. For comparison, the wavelength shift at $1550 \mathrm{~nm}$ of the hightemperature stable type II-IR grating written in $S M F-28$ is also shown. Up to $1000^{\circ} \mathrm{C}$, the Bragg resonances of both the sapphire and $\mathrm{Ge}$-doped silica fibers vary linearly with temperature, $26 \mathrm{pm} /{ }^{\circ} \mathrm{C}$ and $14 \mathrm{pm} /{ }^{\circ} \mathrm{C}$, respectively.

\subsection{Gratings in Other Crystalline Materials}

\subsubsection{Bragg Gratings in Lithium Niobate Waveguides}

Along with sapphire, the femtosecond-IR laser grating inscription technique has also been demonstrated in other crystalline waveguides and fibers. Lithium niobate $\left(\mathrm{LiNbO}_{3}\right)$, because of its ferroelectric nature, is an attractive material for fabrication of active integrated optical devices. The versatility of the waveguides made in this material can be enhanced through the creation of efficient permanent grating structures. Due to the electro-optic properties of the substrate, the lithium niobate Bragg gratings have the potential to satisfy the rapid tuning requirements needed by telecommunication components, such as tunable add/drop filters (ROADMs), tunable dispersion compensators, switches, and so forth that are used for dynamic control of the signal in the network nodes of agile all-optical communication networks.

In our laboratory, gratings were fabricated in $40 \mathrm{~mm}$ long reverse proton exchange (RPE) $\mathrm{LiNbO}_{3}$ channel waveguides using the femtosecond-IR laser-phase mask approach [14]. Using the setup shown in Figure 1 with an $f=30 \mathrm{~mm}$ focusing lens and the $4.28 \mu \mathrm{m}$ pitched phase mask in the twobeam interference regime and $600 \mu \mathrm{J}$ pulse energies, grating responses up to $-3.5 \mathrm{~dB}$ in transmission for exposures at $100 \mathrm{~Hz}$ for 1 minute were achieved. The peak field intensity corresponding to the pulse energies used was 2.0 to $2.5 \times$ $10^{13} \mathrm{~W} / \mathrm{cm}^{2}$, which is of the same order of magnitude as previously established IR ultrafast laser ablation thresholds of $\mathrm{LiNbO}_{3}[62]$. The spectral responses of the grating are shown 


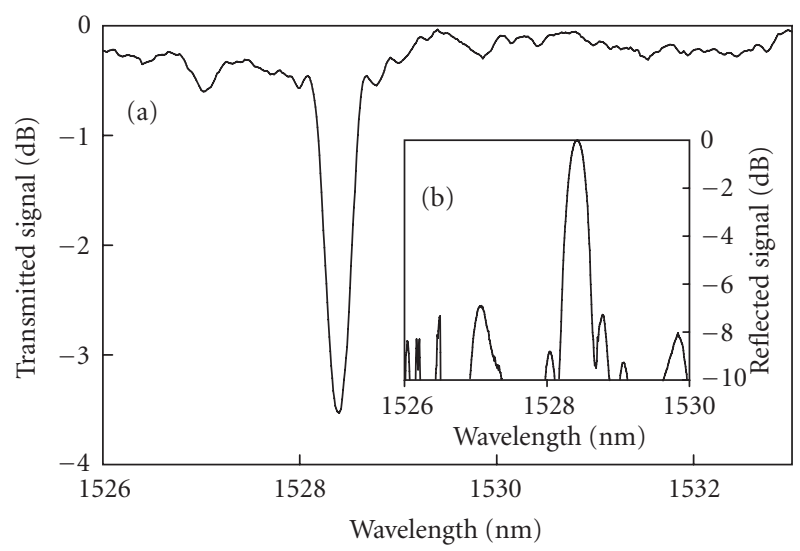

FIgURE 29: (a) Transmitted spectral response of a lithium niobate grating made with femtosecond IR-radiation. (b) Reflected spectral response.

in Figures 29(a) and 29(b). The measured Bragg reflection corresponds to a sixth-order resonance for an effective index of the propagation mode through the waveguide of 2.135.

Bragg gratings made in the $\mathrm{LiNbO}_{3}$ waveguide corresponding to the spectral responses presented in Figure 29 were inspected visually with an optical microscope. As can be seen in Figure 30(a), a surface relief corrugated grating has formed on the waveguide that is a typical ablation effect. Measurement of the surface relief grating with an atomic force microscope revealed a $300 \mathrm{~nm}$ corrugation depth.

In the regions were the ablation process is incomplete, a secondary grating could be seen a few microns below the crystal surface displaying a different physical structure as compared to that of the surface grating (Figure 30(b)). The surface and subsurface gratings are in phase suggesting that they are being created by the same laser exposure. The surface ablation threshold is less than that of the damage threshold to the subsurface material. The core of the waveguide is positioned near the surface and within the Rayleigh range of the $30 \mathrm{~mm}$ focal length lens $(\sim 20 \mu \mathrm{m})$. It is expected therefore that the surface and subsurface regions see the same threshold intensity. The subsurface refractive index change does not have the morphology of catastrophic damage. The surface and subsurface gratings develop simultaneously. Surface grating development ultimately arrests the evolution of the subsurface grating due to scattering of the femtosecondIR radiation by the surface layer.

\subsubsection{YAG Fiber Bragg Grating}

The femtosecond-IR laser phase mask technique was used to inscribe Bragg gratings into $100 \mu \mathrm{m}$ diameter yttrium aluminium garnet (YAG) fiber rod manufactured by MicroMaterials Inc. Using a $4.28-\mu \mathrm{m}$-pitch phase mask and $900 \mu \mathrm{J}$ pulse energies and an $f=30 \mathrm{~mm}$ focal length cylindrical lens, a grating structure was observed under the optical microscope as shown in Figure 31.

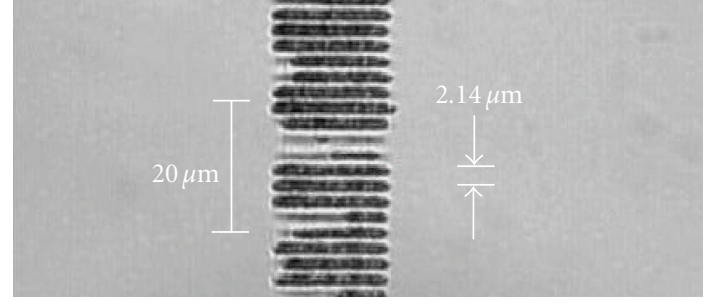

(a)

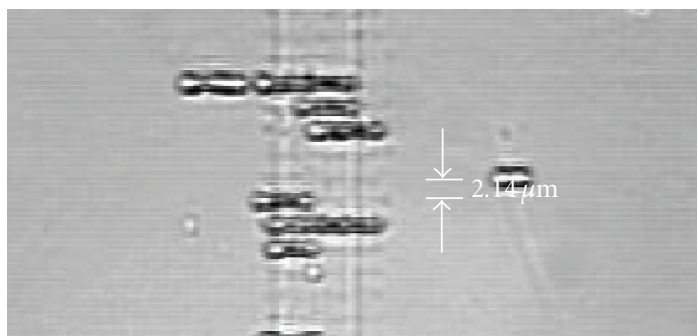

(b)

FIgURE 30: Optical microscope image of the grating induced (a) on the surface and (b) below the surface of the lithium niobate waveguide.

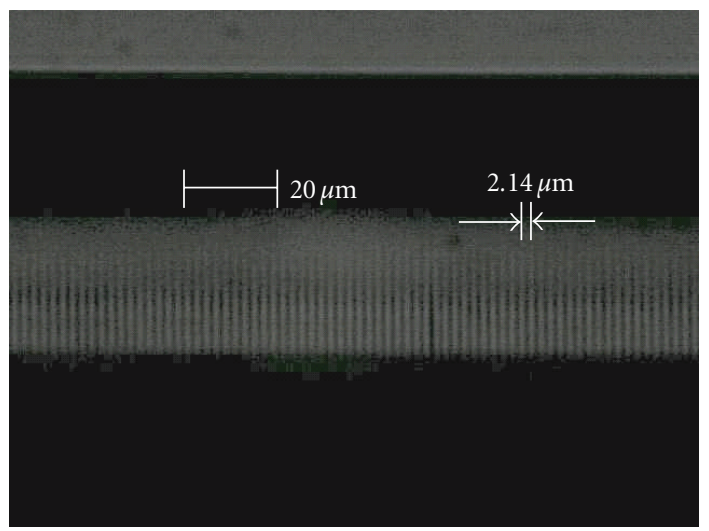

FIGURE 31: Optical microscope image of the grating induced in $100 \mu \mathrm{m}$ diameter YAG fiber.

\section{Conclusions}

In this paper, we have summarized our work on femtosecond laser inscription of Bragg gratings in various materials. The use of high-peak-power femtosecond infrared radiation and a phase mask for the inscription of Bragg gratings in optical fiber and waveguides has been shown to be as easy to implement as standard ultraviolet laser grating inscription techniques. The true advantage of the femtosecond technique over the ultraviolet laser approach is that Bragg gratings can be inscribed in many more optical materials that need not be sensitive to ultraviolet radiation. In fact, to date, we have successfully inscribed grating structures in any solid material that is transparent to the infrared radiation. As a result, not only traditional Bragg grating sensor and telecommunication applications can be realized using our approach but also 
applications where grating inscription was formerly not possible. Some of these applications include grating inscription in exotic waveguide structures such as crystalline sapphire optical fiber and lithium niobate waveguides, glasses such as pure silica, borosilicate, actively doped silica, fluoride, phosphate glasses, and photonic crystal fibers. Depending on the intensity regime used, high spectral quality gratings can be easily manufactured for telecommunication type applications such as dense wavelength division multiplexing or fiber laser cavity mirrors. High reliability gratings can be easily fabricated by direct writing through the standard protective coatings of fibers, removing extra processing steps in grating manufacturing. With higher intensities, high spectral quality gratings can be written that are thermally stable up to the glass transition temperature of the material into which they are written. Such gratings show potential for high temperature sensing applications $\left(1000^{\circ} \mathrm{C}\right)$. Gratings in sapphire fiber could conceivably be used in high-temperature sensor applications up to $2000^{\circ} \mathrm{C}$.

\section{References}

[1] K. M. Davis, K. Miura, N. Sugimoto, and K. Hirao, "Writing waveguides in glass with a femtosecond laser," Optics Letters, vol. 21, no. 21, pp. 1729-1731, 1996.

[2] K.-I. Kawamura, N. Sarukura, M. Hirano, and H. Hosono, "Holographic encoding of fine-pitched micrograting structures in amorphous $\mathrm{SiO}_{2}$ thin films on silicon by a single femtosecond laser pulse," Applied Physics Letters, vol. 78, no. 8, pp. 1038-1040, 2001.

[3] E. Fertein, C. Przygodzki, H. Delbarre, A. Hidayat, M. Douay, and P. Niay, "Refractive-index changes of standard telecommunication fiber through exposure to femto-second laser pulses at $810 \mathrm{~cm}$," Applied Optics, vol. 40, no. 21, pp. 35063508, 2001.

[4] Y. Kondo, K. Nouchi, T. Mitsuyu, M. Watanabe, P. G. Kazansky, and K. Hirao, "Fabrication of long-period fiber gratings by focused irradiation of infrared femtosecond laser pulses," Optics Letters, vol. 24, no. 10, pp. 646-648, 1999.

[5] L. Sudrie, M. Franco, B. Prade, and A. Mysyrowicz, "Study of damage in fused silica induced by ultra-short IR laser pulses," Optics Communications, vol. 191, no. 3-6, pp. 333-339, 2001.

[6] K. O. Hill and G. Meltz, "Fiber Bragg grating technology fundamentals and overview," Journal of Lightwave Technology, vol. 15, no. 8, pp. 1263-1276, 1997.

[7] A. Dragomir, D. N. Nikogosyan, K. A. Zagorulko, P. G. Kryukov, and E. M. Dianov, "Inscription of fiber Bragg gratings by ultraviolet femtosecond radiation," Optics Letters, vol. 28 , no. 22, pp. 2171-2173, 2003.

[8] K. A. Zagorulko, P. G. Kryukov, Y. V. Larionov, et al., "Fabrication of fiber Bragg gratings with $267 \mathrm{~nm}$ femtosecond radiation," Optics Express, vol. 12, no. 24, pp. 5996-6001, 2004.

[9] L. B. Fu, G. D. Marshall, J. A. Bolger, et al., "Femtosecond laser writing Bragg gratings in pure silica photonic crystal fibres," Electronics Letters, vol. 41, no. 11, pp. 638-640, 2005.

[10] G. Violakis, M. Konstantaki, and S. Pissadakis, "Accelerated recording of negative index gratings in Ge-doped optical fibers using 248-nm 500-fs laser radiation," IEEE Photonics Technology Letters, vol. 18, no. 10, pp. 1182-1184, 2006.

[11] S. J. Mihailov, C. W. Smelser, P. Lu, et al., "Fiber Bragg gratings made with a phase mask and 800 -nm femtosecond radiation," Optics Letters, vol. 28, no. 12, pp. 995-997, 2003.
[12] S. J. Mihailov, C. W. Smelser, D. Grobnic, et al., "Bragg gratings written in all- $\mathrm{SiO}_{2}$ and Ge-doped core fibers with 800-nm femtosecond radiation and a phase mask," Journal of Lightwave Technology, vol. 22, no. 1, pp. 94-100, 2004.

[13] D. Grobnic, S. J. Mihailov, C. W. Smelser, M. Becker, and M. W. Rothhardt, "Femtosecond laser fabrication of Bragg gratings in borosilicate ion-exchange waveguides," IEEE Photonics Technology Letters, vol. 18, no. 13, pp. 1403-1405, 2006.

[14] D. Grobnic, S. J. Mihailov, C. W. Smelser, F. Genereux, G. Baldenberger, and R. Vallee, "Bragg gratings made in reverse proton exchange lithium niobate waveguides with a femtosecond IR laser and a phase mask," IEEE Photonics Technology Letters, vol. 17, no. 7, pp. 1453-1455, 2005.

[15] D. Grobnic, S. J. Mihailov, C. W. Smelser, and H. Ding, "Sapphire fiber Bragg grating sensor made using femtosecond laser radiation for ultrahigh temperature applications," IEEE Photonics Technology Letters, vol. 16, no. 11, pp. 2505-2507, 2004.

[16] D. Grobnic, S. J. Mihailov, H. Ding, F. Bilodeau, and C. W. Smelser, "Single and low order mode interrogation of a multimode sapphire fibre Bragg grating sensor with tapered fibres," Measurement Science and Technology, vol. 17, no. 5, pp. 980-984, 2006.

[17] D. Grobnic, S. J. Mihailov, and C. W. Smelser, "Femtosecond IR laser inscription of Bragg gratings in single- and multimode fluoride fibers," IEEE Photonics Technology Letters, vol. 18, no. 24, pp. 2686-2688, 2006.

[18] D. Grobnic, S. J. Mihailov, R. B. Walker, and C. W. Smelser, "Characteristics of strong Bragg gratings made with femtosecond IR radiation in heavily doped $\mathrm{Er}^{3+}$ and $\mathrm{Yb}^{3+}$ silica fibers," in Bragg Gratings, Photosensitivity and Poling in Glass Waveguides (BGPP '07), Quebec City, Canada, September 2007, paper BTuC4.

[19] D. Grobnic, S. J. Mihailov, R. B. Walker, C. W. Smelser, C. Lafond, and A. Croteau, "Bragg gratings made with a femtosecond laser in heavily doped Er-Yb phosphate glass fiber," IEEE Photonics Technology Letters, vol. 19, no. 12, pp. 943-945, 2007.

[20] S. J. Mihailov, D. Grobnic, H. Ding, C. W. Smelser, and J. Broeng, "Femtosecond IR laser fabrication of Bragg gratings in photonic crystal fibers and tapers," IEEE Photonics Technology Letters, vol. 18, no. 17, pp. 1837-1839, 2006.

[21] C. W. Smelser, S. J. Mihailov, D. Grobnic, et al., "Multiplebeam interference patterns in optical fiber generated with ultrafast pulses and a phase mask," Optics Letters, vol. 29, no. 13, pp. 1458-1460, 2004.

[22] C. W. Smelser, D. Grobnic, and S. J. Mihailov, "Generation of pure two-beam interference grating structures in an optical fiber with a femtosecond infrared source and a phase mask," Optics Letters, vol. 29, no. 15, pp. 1730-1732, 2004.

[23] C. W. Smelser, S. J. Mihailov, and D. Grobnic, "Formation of type I-IR and type II-IR gratings with an ultrafast IR laser and a phase mask," Optics Express, vol. 13, no. 14, pp. 5377-5386, 2005.

[24] J. Nishii, N. Kitamura, H. Yamanaka, H. Hosono, and H. Kawazoe, "Ultraviolet-raditationinduced chemical reactions through one- and two- photon absorption processes in $\mathrm{GeO}_{2}$ $\mathrm{SiO}_{2}$ glasses," Optics Letters, vol. 20, no. 10, pp. 1184-1186, 1995.

[25] P. J. Lemaire, R. M. Atkins, V. Mizrahi, and W. A. Reed, "High pressure $\mathrm{H}_{2}$ loading as a technique for achieving ultrahigh UV photosensitivity and thermal sensitivity in $\mathrm{GeO}_{2}$ doped optical fibres," Electronics Letters, vol. 29, no. 13, pp. 1191-1193, 1993. 
[26] T.-E. Tsai, G. M. Williams, and E. J. Friebele, "Index structure of fiber Bragg gratings in $\mathrm{Ge}_{-} \mathrm{SiO}_{2}$ fibers," Optics Letters, vol. 22, no. 4, pp. 224-226, 1997.

[27] C. W. Smelser, S. J. Mihailov, and D. Grobnic, "Hydrogen loading for fiber grating writing with a femtosecond laser and a phase mask," Optics Letters, vol. 29, no. 18, pp. 2127-2129, 2004.

[28] R. Kashyap, Fiber Bragg Gratings, Academic Press, New York, NY, USA, 1999.

[29] L. A. Weller-Brophy and D. G. Hall, "Analysis of waveguide gratings: application of Rouard's method," Journal of the Optical Society of America A, vol. 2, no. 6, pp. 863-871, 1985.

[30] L. A. Weller-Brophy and D. G. Hall, "Analysis of waveguide gratings: a comparison of the results of Rouard's method and coupled-mode theory," Journal of the Optical Society of America A, vol. 4, no. 1, pp. 60-65, 1987.

[31] Z. H. Wang, G.-D. Peng, and P. L. Chu, "Improved Rouard's method for fiber and waveguide gratings," Optics Communications, vol. 177, no. 1-6, pp. 245-250, 2000.

[32] C. W. Smelser, S. J. Mihailov, and D. Grobnic, "Rouard's method modeling of type I-IR fiber Bragg gratings made using an ultrafast IR laser and a phase mask," Journal of the Optical Society of America B, vol. 23, no. 10, pp. 2011-2017, 2006.

[33] W. X. Xie, M. Douay, P. Bernage, P. Niay, J. F. Bayon, and T. Georges, "Second order diffraction efficiency of Bragg gratings written within germanosilicate fibres," Optics Communications, vol. 101, no. 1-2, pp. 85-91, 1993.

[34] D. M. Rayner, A. Naumov, and P. B. Corkum, "Ultrashort pulse non-linear optical absorption in transparent media," Optics Express, vol. 13, no. 9, pp. 3208-3217, 2005.

[35] D. Grobnic, S. J. Mihailov, and C. W. Smelser, "High order spectral response characteristics of fiber Bragg gratings made with ultrafast IR radiation and phase mask," in Proceedings of Bragg Gratings, Poling \& Photosensitivity/ 30th Australian Conference on Optical Fibre Technology (BGPP/ACOFT '05), pp. 463-464, Sydney, Australia, July 2005.

[36] C. W. Smelser, S. J. Mihailov, and D. Grobnic, "Characterization of Fourier components in type I infrared ultrafast laser induced fiber Bragg gratings," Optics Letters, vol. 32, no. 11, pp. 1453-1455, 2007.

[37] T. Erdogan, "Fiber grating spectra," Journal of Lightwave Technology, vol. 15, no. 8, pp. 1277-1294, 1997.

[38] L. Dong, G. Qi, M. Marro, et al., "Suppression of cladding mode coupling loss in fiber Bragg gratings," Journal of Lightwave Technology, vol. 18, no. 11, pp. 1583-1590, 2000.

[39] D. Grobnic, C. W. Smelser, S. J. Mihailov, R. B. Walker, and P. Lu, "Fiber Bragg gratings with suppressed cladding modes made in SMF-28 with a femtosecond IR laser and a phase mask," IEEE Photonics Technology Letters, vol. 16, no. 8, pp. 1864-1866, 2004.

[40] A. Martinez, I. Y. Khrushchev, and I. Bennion, "Direct inscription of Bragg gratings in coated fibers by an infrared femtosecond laser," Optics Letters, vol. 31, no. 11, pp. 16031605, 2006.

[41] S. J. Mihailov, D. Grobnic, and C. W. Smelser, "Efficient grating writing through fibre coating with femtosecond IR radiation and phase mask," Electronics Letters, vol. 43, no. 8, pp. 442-443, 2007.

[42] S. Martin, J. Krüger, A. Hertwig, A. Fiedler, and W. Kautek, "Femtosecond laser interaction with protection materials," Applied Surface Science, vol. 208-209, pp. 333-339, 2003.
[43] S. Baudach, J. Bonse, and W. Kautek, "Ablation experiments on polyimide with femtosecond laser pulses," Applied Physics A, vol. 69, no. 7, pp. S395-S398, 1999.

[44] D. Grobnic, S. J. Mihailov, C. W. Smelser, and R. T. Ramos, "Ultrafast IR laser writing of strong Bragg gratings through the coating of high Ge-doped optical fibers," IEEE Photonics Technology Letters, vol. 20, no. 12, pp. 973-975, 2008.

[45] W. H. Loh, B. N. Samson, L. Dong, G. J. Cowle, and K. $\mathrm{Hsu}$, "High performance single frequency fiber grating-based erbium/ytterbium-codoped fiber lasers," Journal of Lightwave Technology, vol. 16, no. 1, pp. 114-118, 1998.

[46] L. Dong, W. H. Loh, J. E. Caplen, J. D. Minelly, K. Hsu, and L. Reekie, "Efficient single-frequency fiber lasers with novel photosensitive Er/Yb optical fibers," Optics Letters, vol. 22, no. 10, pp. 694-696, 1997.

[47] Y. Lai, A. Martinez, I. Khrushchev, and I. Bennion, "Distributed Bragg reflector fiber laser fabricated by femtosecond laser inscription," Optics Letters, vol. 31, no. 11, pp. 1672-1674, 2006.

[48] E. Wikszak, J. Thomas, J. Burghoff, et al., "Erbium fiber laser based on intracore femtosecond-written fiber Bragg grating," Optics Letters, vol. 31, no. 16, pp. 2390-2392, 2006.

[49] M. Bernier, D. Faucher, R. Vallée, et al., "Bragg gratings photoinduced in ZBLAN fibers by femtosecond pulses at 800 nm," Optics Letters, vol. 32, no. 5, pp. 454-456, 2007.

[50] N. Groothoff, J. Canning, E. Buckley, K. Lyttikainen, and J. Zagari, "Bragg gratings in air-silica structured fibers," Optics Letters, vol. 28, no. 4, pp. 233-235, 2003.

[51] D. Grobnic, H. Ding, S. J. Mihailov, C. W. Smelser, and J. Broeng, "High birefringence fibre Bragg gratings written in tapered photonic crystal fibre with femtosecond IR radiation," Electronics Letters, vol. 43, no. 1, pp. 16-18, 2007.

[52] V. Mizrahi, D. J. DiGiovanni, R. M. Atkins, S. G. Grubb, Y.-K. Park, and J.-M. P. Delavaux, "Stable single-mode erbium fibergrating laser for digital communication," Journal of Lightwave Technology, vol. 11, no. 12, pp. 2021-2025, 1993.

[53] S. Kreger, S. Calvert, and E. Udd, "High pressure sensing using fiber Bragg gratings written in birefringent side hole fiber," in Proceedings of the 15th Optical Fiber Sensors Conference Technical Digest (OFS '02), vol. 1, pp. 355-358, Portland, Ore, USA, May 2002.

[54] T. Erdogan and V. Mizrahi, "Characterization of UV-induced birefringence in photosensitive Ge-doped silica optical fibers," Journal of the Optical Society of America B, vol. 11, no. 10, pp. 2100-2105, 1994.

[55] P. Lu, D. Grobnic, and S. J. Mihailov, "Characterization of the birefringence in fiber Bragg gratings fabricated with an ultrafast-infrared laser," Journal of Lightwave Technology, vol. 25, no. 3, pp. 779-786, 2007.

[56] D. Grobnic, S. J. Mihailov, and C. W. Smelser, "Localized high birefringence induced in $S M F-28$ fiber by femtosecond IR laser exposure of the cladding," Journal of Lightwave Technology, vol. 25, no. 8, pp. 1996-2001, 2007.

[57] A. D. Kersey, M. A. Davis, H. J. Patrick, et al., "Fiber grating sensors," Journal of Lightwave Technology, vol. 15, no. 8, pp. 1442-1463, 1997.

[58] D. Grobnic, C. W. Smelser, S. J. Mihailov, and R. B. Walker, "Long-term thermal stability tests at $100^{\circ} \mathrm{C}$ of silica fibre Bragg gratings made with ultrafast laser radiation," Measurement Science and Technology, vol. 17, no. 5, pp. 1009-1013, 2006.

[59] A. Wang, S. Gollapudi, R. G. May, K. A. Murphy, and R. O. Claus, "Sapphire optical fiber-based interferometer for high 
temperature environmental applications," Smart Materials and Structures, vol. 4, no. 2, pp. 147-151, 1995.

[60] M. G. Xu, J.-L. Archambault, L. Reekie, and J. P. Dakin, "Discrimination between strain and temperature effects using dual-wavelength fibre grating sensors," Electronics Letters, vol. 30, no. 13, pp. 1085-1087, 1994.

[61] J. D. Love, W. M. Henry, W. J. Stewart, R. J. Black, S. Lacroix, and F. Gonthier, "Tapered single-mode fibres and devices. I. Adiabaticity criteria," IEE Proceedings in Optoelectronics, vol. 138, no. 5, pp. 343-354, 1991.

[62] E. A. Stach, V. Radmilovic, D. Deshpande, A. Malshe, D. Alexander, and D. Doerr, "Nanoscale surface and subsurface defects induced in lithium niobate by a femtosecond laser," Applied Physics Letters, vol. 83, no. 21, pp. 4420-4422, 2003. 